\title{
Application of Synchronization to Cooperative Control and Formation Flight of Spacecraft
}

\author{
Soon-Jo Chung* \\ Iowa State University, IA 50011, USA \\ Umair Ahsun, ${ }^{\dagger}$ Jean-Jacques E. Slotine, ${ }^{\ddagger}$ and David W. Miller ${ }^{\S}$ \\ Massachusetts Institute of Technology, MA 02139, USA
}

\begin{abstract}
This article presents a simple synchronization framework with application to synchronization control of formation flying spacecraft. A dynamical network of multiple Lagrangian systems is constructed by adding diffusive couplings to otherwise freely flying spacecraft. The proposed tracking control law synchronizes an arbitrary number of spacecraft into a common time-varying trajectory with global exponential convergence. The proposed strategy is much simpler than earlier work in terms of both the computational load and the required signals. Furthermore, in contrast with prior work which used simple double integrator models, the proposed method permits highly nonlinear systems and is further extended to adaptive synchronization, partial-state coupling, and time-delayed communications.
\end{abstract}

\section{Introduction}

Motivated by distributed computation and cooperation, abundant in both biological systems (e.g., fish swarms) and artificial machines (e.g., parallel computers), formation flying spacecraft has been a key research topic among many recent advancements. Multiple apertures flying in precise formation are expected to provide unprecedented image resolution, both for astronomy and reconnaissance, ${ }^{4}$ as well as unparalleled reconfigurability. However, many significant technical challenges must be overcome before formation flying interferometers can be realized (see two representative missions in Figure 1). For instance, formation flight requires extensive technology development for precise attitude and position maintenance of multiple spacecraft.

The objective of this paper is to introduce a unified synchronization framework that can be directly applied to the position and attitude synchronization, and cooperative control of formation flight networks, comprised of either identical or heterogenous spacecraft. In this article, synchronization is defined as a complete match of all configuration variables of each dynamical system such that $\mathbf{x}_{1}=\mathbf{x}_{2}=\cdots=\mathbf{x}_{p}$ and $p$ denotes the number of sub-systems in the network. For example, a large number of spacecraft can first synchronize their attitudes and positions to form a certain formation pattern, then track the common position and attitude trajectory to accomplish the given mission. A stellar formation flight interferometer ${ }^{5}$ depends on precision control of relative spacecraft motions, indispensable for coherent interferometric beam combination (Figure 1). The proposed synchronization tracking control law can be implemented for such purposes, thereby achieving more efficient and robust performance through local interactions.

A recent review paper ${ }^{34}$ highlighted the three mains areas for future research that have not been thoroughly addressed in the spacecraft formation flight literature: (1) rigorous stability conditions for cyclic and behavioral architectures, ${ }^{34}$ (2) reduced algorithmic information requirements, and (3) increased robustness/autonomy. While this paper does not provide complete solutions to these three challenges, the unique contributions of this paper are categorized by the aforementioned areas, as follows.

\footnotetext{
*Assistant Professor of Aerospace Engineering, AIAA Member, sjchung@alum.mit.edu.

${ }^{\dagger}$ Research Affiliate, MIT Space Systems Laboratory, umair@alum.mit.edu.

¥Professor of Mechanical Engineering \& Information Sciences, Professor of Brain \& Cognitive Sciences, jjs@mit.edu.

$\S$ Professor, Department of Aeronautics and Astronautics, and AIAA Senior Member, millerd@mit.edu.
} 


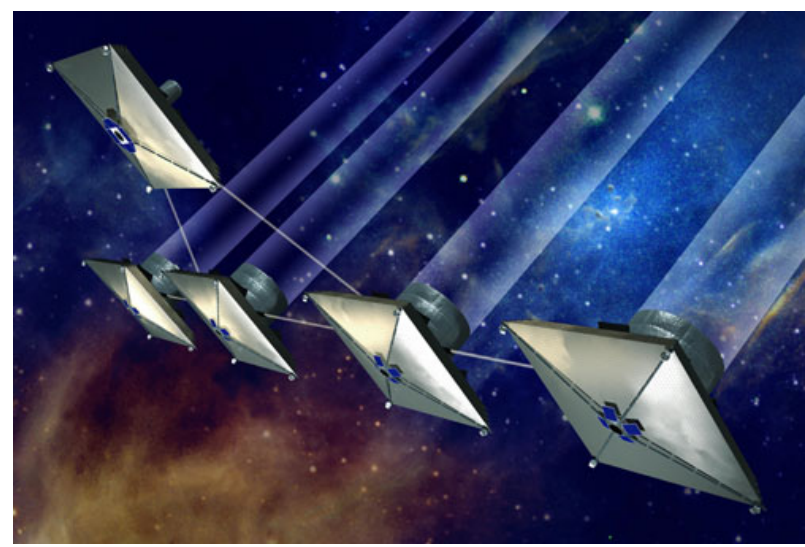

(a) NASA formation flying interferometer TPF, courtesy: NASA, http://planetquest.jpl.nasa.gov

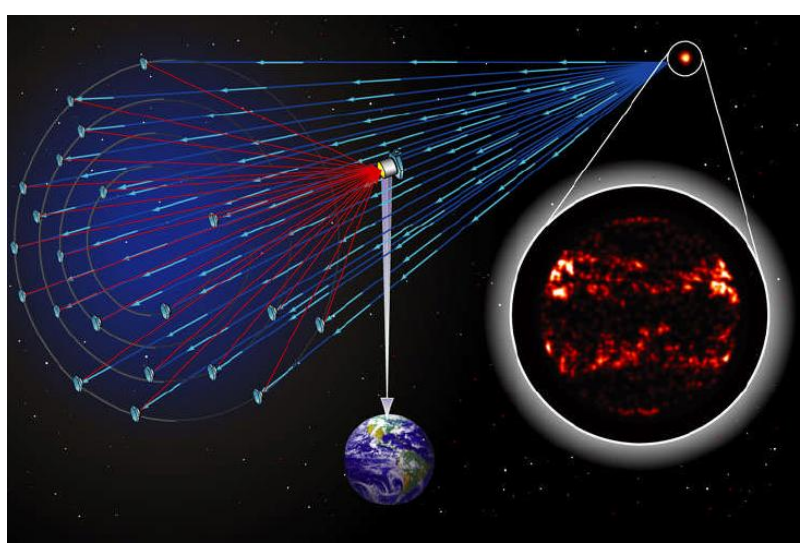

(b) NASA Stellar Imager (SI), courtesy: NASA, http://hires.gsfc.nasa.gov/si

Figure 1. Representative future NASA missions using formation flying spacecraft.

\section{Rigorous Stability Condition for Highly Nonlinear Time Varying Systems}

Prior work on consensus and flocking problems using graphs, particularly popular in the robotics research community, tends to assume very simple dynamics such as double integrators. While such work can be generally applied to the synchronous position control problem of deep space spacecraft formations, the proposed strategy in this paper primarily deals with complex dynamical networks consisting of highly nonlinear time-varying dynamics that are controlled to track a time-varying reference trajectory or leader. It should be noted that determining stability of nonlinear dynamic network systems is much more involved and complex. Many mechanical systems exhibit nonlinear dynamics that cannot be captured by linearization. One might argue that most space systems are not required to follow demanding time-varying trajectories, thus validating linearization or linear coupling control laws. However, there is increased interest in highly agile imaging spacecraft ${ }^{19}$ that undergo wide and rapid slew angle changes. Moreover, in the context of nonlinear control theory, the asymptotic convergence of linear control, employed to stabilize nonlinear systems, may not be sufficient for demanding future mission requirements. In essence, ensuring exponential tracking stability for nonlinear systems is made possible only through nonlinear control.

We introduce contraction analysis ${ }^{14,30,37}$ as our main nonlinear stability tool for reducing the complexity and dimensionality associated with multi-agent systems, thereby deriving exact and global results with exponential convergence with respect to arbitrary time-varying inputs (see the Appendix for the further treatment of contraction theory).

\section{Reduced Information Network}

Another benefit of synchronization is its implication for model reduction. The exponential synchronization of multiple nonlinear dynamics allows us to reduce the dimensionality of the stability analysis of a large network. The model reduction aspect of synchronization, also introduced for spatially inter-connected systems in Ref. 5, is further generalized and strengthened in this article. This implies that once the network is proven to synchronize, we can regard a network as a single set of synchronized dynamics, which simplifies any additional stability analysis. As shall be seen later in the subsequent sections, this model reduction has to do with the fact that there are two time-scales associated with the coupled nonlinear dynamics.

In addition, the proposed control laws are of a decentralized form requiring only local velocity/position coupling feedback for global exponential convergence, thereby facilitating implementation in real systems. As opposed to some previous work using all-to-all coupling ${ }^{29}$ or depending only on tracking the same leader (reference) spacecraft without local interactions, ${ }^{27,36}$ our proposed approach will not only reduce communication burdens, but also increase the overall performance of relative formation flight through local interactions. Further, we mathematically prove that the synchronized maneuvers are also achieved by sharing partial state information. This partial degrees-of-freedom coupling will further reduce the amount of formation state information needed for formation flight. 


\section{Robustness Issues}

We also show that the proposed decentralized control law possesses a property of robustness to interspacecraft time-delays and bounded vanishing disturbances. An adaptive version of the proposed control law is also presented to deal with parametric uncertainties of dynamic models. We briefly review and compare prior work with our proposed approach in the next section.

\section{A. Comparison with Prior Work}

An excellent review on the state-of-the-art of guidance and control techniques for formation flying spacecraft is offered in Ref. 33,34. Some prior work on attitude synchronization rely on tracking a common reference (leader) spacecraft without interactions with local neighbors. Local coupling control laws are suggested in Ref. 10, but individual spacecraft are synchronized to a constant state, thus not permitting an arbitrary reference trajectory. This drawback is particularly true of the consensus problems on graph. ${ }^{23}$ The consensus and the coordination of multi-agent systems ${ }^{8,24,25}$ are closely related with the synchronization problem. In particular, the use of graph theory and Laplacian produced many interesting results. ${ }^{8,12,16,23}$ The main drawback of the aforementioned works is that they mainly deal with very simple dynamic models such as linear systems and single or double integrator models with a constant inertia matrix. Hence, most of earlier work on multi-agent coordination cannot be used for highly nonlinear systems (e.g. helicopters, attitude dynamics of spacecraft, walking robots and manipulator robots). As shall be seen later, the proof of the synchronization for network systems that possess a nonlinear inertia matrix is much more involved and difficult. The present paper focuses on dynamical networks consisting of highly nonlinear systems.

Another representative work ${ }^{29}$ proposes a nonlinear tracking control law to exponentially synchronize multiple robot manipulators in order to track a common desired trajectory. In essence, Ref. 29 proves the stability of the control law with estimated velocity and acceleration states from a set of nonlinear observers. The following difficulties can be identified. The number of variables to be estimated increases with the number of robots to be synchronized, which imposes a significant communication burden. Additionally, the feedback of estimated acceleration errors requires unnecessary information and complexity. Thus, a method to eliminate both the all-to-all coupling and the feedback of the acceleration terms is explored in this paper.

Another notable approach to synchronization of robot networks is to exploit the passivity of the inputoutput dynamics. $^{2,10}$ Its property of robustness to time-delays is particularly attractive. However, robot dynamics are passive only with velocity outputs unless composite variables are employed. In addition, the mutual synchronization problem, which not only synchronizes the sub-members but also enforces them to follow a common reference trajectory, is not addressed. Another recent work using the passive decomposition $^{11,12}$ is interesting in the sense that it shares the same philosophy of mutual synchronization. The passive decomposition describes a strategy of decoupling into two dynamics: the same system representing the internal group formation shape, and the locked system describing the total group maneuver. One drawback of Ref. 11 is its dependency on a centralized control architecture; the decoupling is not generally ensured under the decentralized control. Ref. 12 only considers linear double integrator models, which can degenerate into a trivial problem (see Section IV). We believe our approach using contraction analysis has a clear advantage in its broad applications to a larger class of identical or nonidentical nonlinear systems even with time-delays, non-passive input-output, and complex coupling geometry including partial degrees-of-freedom coupling, while ensuring a simple decentralized coupling control law (see Figure 2 for network structures permitted here).

\section{B. Organization}

The organization of this paper is as follows: dynamic modeling of spacecraft based on the Lagrangian formulation is described in Section II. Section III summarizes the main theorems of this paper; the new tracking control law, which synchronizes each robot to track the same desired trajectory, is proposed. The proof of exponential synchronization is more involved than that of the tracking stability and treated separately in Section IV. The remainder of the paper further highlights the unique contributions of this work. A few examples of dynamics networks are given in Section $\mathrm{V}$ for validating the effectiveness of the proposed synchronization framework. The same section also introduces the partial state couplings. Additionally, the properties of robustness to transmission delays and disturbances are discussed in Section VI. An adaptive control version of the proposed synchronization strategy is also presented in Section VI-C. 


\section{Lagrangian Formulation of Formation Flying Spacecraft}

Our previous work ${ }^{6,7}$ on the synchronization of multiple robots is devoted to the use of the Lagrangian formulation for its simplicity in dealing with complex systems involving multiple dynamics. We show herein that the rotational maneuvers of a rigid spacecraft can be written in this Lagrangian form, thereby permitting direct application of the proposed synchronization strategy ${ }^{6,7}$ to the rotational dynamics of multiple spacecraft. Without loss of generality, the proposed control law can be applied to the position synchronization of formation flying spacecraft, or more generally to the coupled translational and attitude dynamics.

\section{A. Lagrangian Formulation}

The equations of motion for a robot or spacecraft with multiple degrees-of-freedom $\left(\mathbf{q}_{i} \in \mathbb{R}^{n}\right)$ can be derived by exploiting the Euler-Lagrange equations:

$$
L_{i}\left(\mathbf{q}_{i}, \dot{\mathbf{q}}_{i}\right)=\frac{1}{2} \dot{\mathbf{q}}_{i}^{T} \mathbf{M}_{i}\left(\mathbf{q}_{i}\right) \dot{\mathbf{q}}_{i}-V_{i}\left(\mathbf{q}_{i}\right), \quad \frac{d}{d t} \frac{\partial L_{i}\left(\mathbf{q}_{i}, \dot{\mathbf{q}}_{i}\right)}{\partial \dot{\mathbf{q}}_{i}}-\frac{\partial L_{i}\left(\mathbf{q}_{i}, \dot{\mathbf{q}}_{i}\right)}{\partial \mathbf{q}_{i}}=\tau_{i}
$$

where $i,(1 \leq i \leq p)$ denotes the index of spacecraft comprising a spacecraft formation flight network, and $p$ is the total number of the individual elements. Equation (1) can be represented as

$$
\mathbf{M}_{i}\left(\mathbf{q}_{i}\right) \ddot{\mathbf{q}}_{i}+\mathbf{C}_{i}\left(\mathbf{q}_{i}, \dot{\mathbf{q}}_{i}\right) \dot{\mathbf{q}}_{i}+\mathbf{g}_{i}\left(\mathbf{q}_{i}\right)=\tau_{i}
$$

where $\mathbf{g}_{i}\left(\mathbf{q}_{i}\right)=\frac{\partial V}{\mathbf{q}_{i}}$, and, $\tau_{i}$ is a generalized force or torque acting on the $i$-th spacecraft.

It should be emphasized that, among many possible choices, the $\mathbf{C}$ matrix is defined as

$$
c_{i j}=\frac{1}{2} \sum_{k=1}^{n} \frac{\partial M_{i j}}{\partial q_{k}} \dot{q}_{k}+\frac{1}{2} \sum_{k=1}^{n}\left(\frac{\partial M_{i k}}{\partial q_{j}}-\frac{\partial M_{j k}}{\partial q_{i}}\right) \dot{q_{k}}
$$

Then, it is straightforward to show that $\left(\dot{\mathbf{M}}_{i}-2 \mathbf{C}_{i}\right)$ is skew-symmetric, resulting in

$$
\mathbf{x}^{T}\left(\dot{\mathbf{M}}_{i}-2 \mathbf{C}_{i}\right) \mathbf{x}=0, \quad 1 \leq i \leq p
$$

for an arbitrary $\mathbf{x} \in \mathbb{R}^{n}$. This skew-symmetric property can be viewed as a matrix expression of energy conservation, which can also be explained in the context of the passivity formalism. ${ }^{18}$ In the remainder of this paper, the property in (4) is extensively exploited for stability analysis and control synthesis using contraction theory. ${ }^{7}$

We assume that the spacecraft system in (2) is fully actuated. In other words, the number of control inputs is equal to the dimension of their configuration manifold $(n)$.

\section{B. Attitude Dynamics of Rigid Spacecraft}

The aim of the present section is to show that we can establish a Lagrangian dynamics form given in (2) from the rotational attitude dynamics of a rigid spacecraft $(n=3)$. We improve the approach in Ref. 17 in two aspects. First, we introduce the generalized form to explore the increasing interest in agile imaging spacecraft using Control Moment Gyroscope (CMG) ${ }^{19}$ and Variable Speed Control Moment Gyroscope (VSCMG). ${ }^{20}$ Second, we incorporate the Modified Rodrigues Parameters (MRPs), ${ }^{21,22}$ in lieu of the Rodrigues parameters, to overcome the singularity problem at the rotation of $\pm 180 \mathrm{deg}$.

Using the Euler rotational equations of motion, the following equation describes the angular velocity vector $\omega \in \mathbb{R}^{3}$ of the spacecraft in its body axes:

$$
\mathbf{J}_{\mathbf{s} / \mathbf{c}} \dot{\omega}-\left(\mathbf{J}_{\mathbf{s} / \mathbf{c}} \omega\right) \times \omega=\mathbf{u}+\mathbf{d}_{\mathbf{e x t}}
$$

where the internal control torque $\mathbf{u}$, generated either by VSCMGs or CMGs, is defined as ${ }^{19}$

$$
\mathbf{u}=-\dot{\mathbf{h}}+\mathbf{h} \times \omega
$$

Based on (6), a suitable algorithm, such as the pseudo-inverse steering logic ${ }^{19}$ can determine the gimbal angle vector $\gamma$ once the control input $\mathbf{u}$ is computed from the attitude control law. 
Note that the matrix $\mathbf{J}_{\mathbf{s} / \mathbf{c}}$ is the total moment of inertia of the spacecraft, expressed in its body frame, and is symmetric positive definite. Also, $\mathbf{h}$ and $\mathbf{d}_{\mathbf{e x t}}$, all expressed in the spacecraft body-fixed frame, denote the total control momentum vector by CMGs, and the external disturbance torque such as the aerodynamic drag torque and the gravity gradient torque. We also assume that the change of $\mathbf{J}_{\mathbf{s} / \mathbf{c}}$ due to the CMG gimbal angular rate is small (i.e., $\dot{\mathbf{J}}_{\mathbf{s} / \mathbf{c}}=0$ ).

In the case of VSCMGs, the control momentum vector $(\mathbf{h})$ and its rate $(\dot{\mathbf{h}})$ can be written $a^{20}$

$$
\begin{aligned}
\mathbf{h} & =\mathbf{A}_{g} \mathbf{I}_{g} \dot{\gamma}+\mathbf{A}_{s} \mathbf{I}_{w} \boldsymbol{\Omega} \\
\dot{\mathbf{h}} & =\mathbf{A}_{g} \mathbf{I}_{g} \ddot{\gamma}+\mathbf{A}_{s} \mathbf{I}_{w} \dot{\boldsymbol{\Omega}}+\mathbf{A}_{t} \mathbf{I}_{w} \operatorname{diag}(\dot{\boldsymbol{\Omega}}) \dot{\gamma}
\end{aligned}
$$

where $\gamma$ and $\boldsymbol{\Omega}$ denote the gimbal angles and wheel speeds of the VSCMGs, respectively, while $\mathbf{I}_{g}$ and $\mathbf{I}_{w}$ are the moment of inertia of the gimbal structure and the wheel. Also, $\mathbf{A}_{g}, \mathbf{A}_{s}$, and $\mathbf{A}_{t}$ denote the transformation matrices associated with the body-frame representation. ${ }^{20}$

The purpose of introducing (7) is to show that (5) may represent either the rotational dynamics of spacecraft with fixed-speed CMGs (constant $\boldsymbol{\Omega}$ ) or reaction wheels $(\dot{\gamma}=0)$.

To avoid the singularity problem of the Euler angular representation, it is often preferred to use quaternions to represent an angular orientation between two different coordinate frames:

$$
\beta_{1}=e_{1} \sin \frac{\theta}{2}, \quad \beta_{2}=e_{2} \sin \frac{\theta}{2}, \quad \beta_{3}=e_{3} \sin \frac{\theta}{2}, \quad \beta_{4}=\cos \frac{\theta}{2}
$$

where $\mathbf{e}=\left(e_{1}, e_{2}, e_{3}\right)^{T}$ is the Euler axis of rotation expressed in the body frame and $\theta$ is the rotation angle about e.

The modified Rodrigues parameters can be written as: ${ }^{21,22}$

$$
\mathbf{q}=\left(q_{1}, q_{2}, q_{3}\right)^{T}=\mathbf{e} \tan \frac{\theta}{4}
$$

Then, the attitude of the spacecraft has the following relation:

$$
\dot{\mathbf{q}}=\mathbf{Z}(\mathbf{q}) \omega
$$

where

$$
\begin{aligned}
\mathbf{Z}(\mathbf{q}) & =\frac{1}{2}\left(\mathbf{I}\left(\frac{1-\mathbf{q}^{T} \mathbf{q}}{2}\right)+\mathbf{q q}^{T}+\mathbf{S}(\mathbf{q})\right) \\
& =\frac{1}{4}\left[\begin{array}{ccc}
\left(1+{q_{1}}^{2}-{q_{2}}^{2}-{q_{3}}^{2}\right) & 2\left(q_{1} q_{2}-q_{3}\right) & 2\left(q_{1} q_{3}+q_{2}\right) \\
2\left(q_{2} q_{1}+q_{3}\right) & \left(1-q_{1}{ }^{2}+q_{2}{ }^{2}-q_{3}{ }^{2}\right) & 2\left(q_{2} q_{3}-q_{1}\right) \\
2\left(q_{3} q_{1}-q_{2}\right) & 2\left(q_{3} q_{2}+q_{1}\right) & \left(1-q_{1}{ }^{2}-q_{2}{ }^{2}+q_{3}{ }^{2}\right)
\end{array}\right],
\end{aligned}
$$

and the skew-symmetric matrix function, $\mathbf{S}(\mathbf{x})$ for an arbitrary $\mathbf{x} \in \mathbb{R}^{3}$ is defined as

$$
\mathbf{S}(\mathbf{x})=\left[\begin{array}{ccc}
0 & -x_{3} & x_{2} \\
x_{3} & 0 & -x_{1} \\
-x_{2} & x_{1} & 0
\end{array}\right]
$$

Also, note that the corresponding quaternions in (8) can be obtained from the modified Rodrigues parameters by the following transformation

$$
\beta_{i}=2 q_{i} /\left(1+\mathbf{q}^{T} \mathbf{q}\right), \quad i=1,2,3, \quad \beta_{4}=\left(1-\mathbf{q}^{T} \mathbf{q}\right) /\left(1+\mathbf{q}^{T} \mathbf{q}\right),
$$

while its inverse transformation can be written

$$
q_{i}=\beta_{i} /\left(1+\beta_{4}\right), \quad i=1,2,3
$$

By combining (5) and (10), the following equations of motion are obtained with respect to q:

$$
\mathbf{M}(\mathbf{q}) \ddot{\mathbf{q}}+\mathbf{C}(\mathbf{q}, \dot{\mathbf{q}}) \dot{\mathbf{q}}=\tau+\tau_{\text {ext }}
$$


where

$$
\begin{aligned}
\tau & =\mathbf{Z}^{-T} \mathbf{u}, \quad \tau_{\text {ext }}=\mathbf{Z}^{-T} \mathbf{d}_{\mathbf{e x t}} \\
\mathbf{M}(\mathbf{q}) & =\mathbf{Z}^{-T} \mathbf{J}_{\mathbf{s} / \mathbf{c}} \mathbf{Z}^{-1} \\
\mathbf{C}(\mathbf{q}, \dot{\mathbf{q}}) & =-\mathbf{Z}^{-T} \mathbf{J}_{\mathbf{s} / \mathbf{c}} \mathbf{Z}^{-1} \dot{\mathbf{Z}} \mathbf{Z}^{-1}-\mathbf{Z}^{-T} \mathbf{S}\left(\mathbf{J}_{\mathbf{s} / \mathbf{c}} \omega\right) \mathbf{Z}^{-1}
\end{aligned}
$$

Also, note that $\mathbf{S}\left(\mathbf{J}_{\mathbf{s} / \mathbf{c}} \omega\right)=\mathbf{S}\left(\mathbf{J}_{\mathbf{s} / \mathbf{c}} \mathbf{Z}^{-1} \dot{\mathbf{q}}\right)$ from (10). It should be emphasized that we should not cancel the common term $\mathbf{Z}^{-T}$ of (15), because it would result in a non-symmetric $\mathbf{M}(\mathbf{q})$. In essence, we established a Lagrangian formulation for the attitude dynamics of rigid spacecraft. This allows us to apply a wealth of nonlinear control laws to spacecraft dynamics, including the proposed control strategy in Ref. 6 , that were originally developed for robot dynamics. As discussed in (4), the most important feature of (15) is to have a skew-symmetric $\dot{\mathbf{M}}-2 \mathbf{C}$ due to energy conservation. Indeed, we can verify that

$$
\dot{\mathbf{M}}-2 \mathbf{C}=\frac{d \mathbf{Z}^{-T}}{d t} \mathbf{J}_{\mathbf{s} / \mathbf{c}} \mathbf{Z}^{-1}-\mathbf{Z}^{-T} \mathbf{J}_{\mathbf{s} / \mathbf{c}} \frac{d \mathbf{Z}^{-1}}{d t}+2 \mathbf{Z}^{-T} \mathbf{S}\left(\mathbf{J}_{\mathbf{s} / \mathbf{c}} \omega\right) \mathbf{Z}^{-1}
$$

is skew-symmetric, which follows the fact that $\mathbf{S}\left(\mathbf{J}_{\mathbf{s} / \mathbf{c}} \omega\right)$ is skew-symmetric. Without loss of generality, the control torque $\mathbf{u}$ generated by momentum wheels $(\dot{\gamma}=0)$ can be defined as $\mathbf{u}=-\dot{\mathbf{h}}$. Then, the $\mathbf{S}\left(\mathbf{J}_{\mathbf{s} / \mathrm{c}} \omega\right)$ in (16) is replaced by $\mathbf{S}\left(\mathbf{J}_{\mathbf{s} / \mathbf{c}} \omega+\mathbf{h}\right)$ in order to account for the gyro stiffening effect of the wheels.

In the subsequent sections, the rotational dynamics formulation in (15) is used to develop a nonlinear synchronization tracking control law for multiple formation flying spacecraft.

\section{Translational Dynamics}

For deep space formation flight, the translational dynamics can be essentially modeled as double integrators, ${ }^{33}$ which can easily be augmented with the attitude dynamics in (15). Alternatively, similar to Ref. 27, 28, the coupled translational and rotational motions of formation spacecraft can be written in the Lagrangian form in (2). Then, the proposed decentralized tracking control can be effectively applied without loss of generality. For the translational dynamics, synchronization corresponds to $\mathbf{y}_{1}=\mathbf{y}_{2}=\cdots=\mathbf{y}_{p}$ where $\mathbf{y}_{i}, 1 \leq i \leq p$ connotes a vector of biased variables constructed from the configuration vector $\mathbf{x}_{i}$ such that $\mathbf{x}_{i}(t)=\mathbf{y}_{i}(t)+\mathbf{b}_{i}(t)$ and the separation vector $\mathbf{b}_{i}(t)$ is independent of the dynamics. ${ }^{7}$ Alternatively, each position vector $\mathbf{x}_{i}$ can be defined from different origins. While the synchronization of multiple double integrators can be adequately studied by utilizing prior work on consensus ${ }^{23}$ and leader-follower problem, ${ }^{1,13}$ this paper attempts to bridge the technological gap associated with highly nonlinear systems such as the attitude dynamics of spacecraft given in (15).

\section{Decentralized Nonlinear Synchronization Control}

We consider the attitude synchronization of multiple spacecraft following a common time-varying trajectory. A tracking controller introduced in this section achieves not only global and exponential synchronization of the configuration variables, but also global exponential convergence to the desired trajectory.

\section{A. Proposed Synchronization Control Strategy}

The following decentralized tracking control law with two-way-ring symmetry is proposed for the $i$-th robot in the network comprised of $p$ spacecraft (see Figure 2(a)):

$$
\tau_{i}=\mathbf{M}_{i}\left(\mathbf{q}_{i}\right) \ddot{\mathbf{q}}_{i r}+\mathbf{C}_{i}\left(\mathbf{q}_{i}, \dot{\mathbf{q}}_{i}\right) \dot{\mathbf{q}}_{i r}+\mathbf{g}\left(\mathbf{q}_{i}\right)-\mathbf{K}_{1} \mathbf{s}_{i}+\mathbf{K}_{2} \mathbf{s}_{i-1}+\mathbf{K}_{2} \mathbf{s}_{i+1}
$$

where a positive-definite matrix $\mathbf{K}_{1} \in \mathbb{R}^{n \times n}$ is a feedback gain for the $i$-th robot, and another positive-definite matrix $\mathbf{K}_{2} \in \mathbb{R}^{n \times n}$ is a coupling gain with the adjacent members $(i-1$ and $i+1)$. For two-spacecraft networks, the last coupling term with the $i+1$-th member in (18) is not used. Also, $\dot{\mathbf{q}}_{i r}$ and $\mathbf{s}_{i}$ are defined such that

$$
\dot{\mathbf{q}}_{i r}=\dot{\mathbf{q}}_{d}+\Lambda\left(\mathbf{q}_{d}-\mathbf{q}_{i}\right), \quad \mathbf{s}_{i}=\dot{\mathbf{q}}_{i}-\dot{\mathbf{q}}_{i r}=\dot{\mathbf{q}}_{i}-\dot{\mathbf{q}}_{d}+\boldsymbol{\Lambda}\left(\mathbf{q}_{i}-\mathbf{q}_{d}\right)
$$

where $\boldsymbol{\Lambda}$ is a positive diagonal matrix. The time-varying desired trajectory $\mathbf{q}_{d}$ can be a formation flying guidance command or the trajectory of a leader spacecraft. 


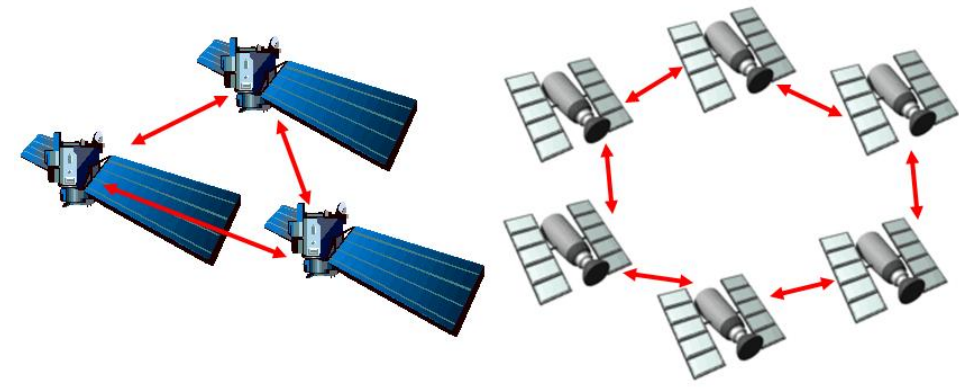

(a) identical spacecraft

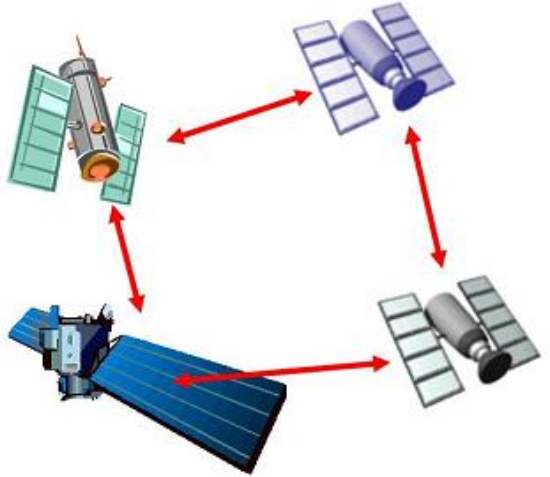

(b) non-identical spacecraft

Figure 2. Multi-agent networks of identical or nonidentical spacecraft using local couplings. They are on balanced bi-directional graphs, but a more complex geometry or leader-follower network can also be constructed. ${ }^{6}$

It should be noted again that one of the main contributions of this paper lies with the use of a new differential framework, yielding the exact proof of nonlinear stability under a variety of conditions, while many others can still come up with nonlinear control laws, similar to (18), without a rigorous stability proof. Note that the above control law requires only the coupling feedback of the most adjacent spacecraft $(i-1$ and $i+1)$ for exponential convergence (see Figure 2). Consequently, the last $(p$-th) robot is connected with the first robot to form a ring network as suggested in Ref. 37. In order to construct more a complex geometry rather than a ring network, concurrent synchronization ${ }^{26}$ can be used, as expanded upon in Ref. 7 .

Let us define the following $p \times p$ block matrices:

$$
\left[\mathbf{L}_{\mathbf{A}, \mathbf{B}}^{p}\right]=\left[\begin{array}{cccccc}
\mathbf{A} & \mathbf{B} & \mathbf{0} & \mathbf{0} & \cdots & \mathbf{B} \\
\mathbf{B} & \mathbf{A} & \mathbf{B} & \mathbf{0} & \cdots & \mathbf{0} \\
\vdots & \ddots & \ddots & \ddots & & \vdots \\
\mathbf{0} & & \mathbf{B} & \mathbf{A} & \mathbf{B} & \mathbf{0} \\
\mathbf{B} & \cdots & \mathbf{0} & \mathbf{0} & \mathbf{B} & \mathbf{A}
\end{array}\right]_{p \times p}, \quad\left[\mathbf{U}_{\mathbf{A}}^{p}\right]=\left[\begin{array}{cccc}
\mathbf{A} & \mathbf{A} & \cdots & \mathbf{A} \\
\mathbf{A} & \mathbf{A} & \cdots & \mathbf{A} \\
\vdots & \vdots & \ddots & \vdots \\
\mathbf{A} & \mathbf{A} & \cdots & \mathbf{A}
\end{array}\right]_{p \times p}
$$

By the definition of the controller in (18), $\left[\mathbf{L}_{\mathbf{A}, \mathbf{B}}^{p}\right]$ has only three nonzero matrix elements in each row (i.e., $\mathbf{A}, \mathbf{B}, \mathbf{B})$.

Then, the closed-loop dynamics for the whole formation, by using (2) and (18), can be written as

$$
[\mathbf{M}] \dot{\mathbf{x}}+[\mathbf{C}] \mathbf{x}+\left(\left[\mathbf{L}_{\mathbf{K}_{1},-\mathbf{K}_{2}}^{p}\right]+\left[\mathbf{U}_{\mathbf{K}_{2}}^{p}\right]\right) \mathbf{x}=\left[\mathbf{U}_{\mathbf{K}_{2}}^{p}\right] \mathbf{x}
$$

where

$$
[\mathbf{M}]=\left[\begin{array}{ccc}
\mathbf{M}_{1}\left(\mathbf{q}_{1}\right) & \cdots & \mathbf{0} \\
\vdots & \ddots & \vdots \\
\mathbf{0} & \cdots & \mathbf{M}_{p}\left(\mathbf{q}_{p}\right)
\end{array}\right], \quad[\mathbf{C}]=\left[\begin{array}{ccc}
\mathbf{C}_{1}\left(\mathbf{q}_{1}, \dot{\mathbf{q}}_{1}\right) & \cdots & \mathbf{0} \\
\vdots & \ddots & \vdots \\
\mathbf{0} & \cdots & \mathbf{C}_{p}\left(\mathbf{q}_{p}, \dot{\mathbf{q}}_{p}\right)
\end{array}\right], \quad \mathbf{x}=\left(\begin{array}{c}
\mathbf{s}_{1} \\
\vdots \\
\mathbf{s}_{p}
\end{array}\right)
$$

Note that $\left[\mathbf{L}_{\mathbf{K}_{1},-\mathbf{K}_{2}}^{p}\right]$ can be viewed as the weighted Laplacian of the network in the context of graph theory. In other words, $\left[\mathbf{L}_{\mathbf{K}_{1},-\mathbf{K}_{2}}^{p}\right]$ indicates the connectivity with adjacent systems as well as the strength of the coupling by $\mathbf{K}_{2}$. Note that there are only three nonzero elements in each row of the matrix, which implies that there exist diffusive couplings only between adjacent members. The network graphs illustrated in Figure 2 are balanced due to bi-directional coupling. ${ }^{23}$ However, it should be noted that the matrix $\left[\mathbf{L}_{\mathbf{K}_{1},-\mathbf{K}_{2}}^{p}\right]$ is different from the standard Laplacian found in Ref. 23. By definition, every row sum of the Laplacian matrix is zero. Hence, the Laplacian matrix always has a zero eigenvalue corresponding to a right eigenvector, $\mathbf{1}=(1,1, \cdots, 1)^{T} \cdot{ }^{23}$ In contrast, a strictly positive definite $\left[\mathbf{L}_{\mathbf{K}_{1},-\mathbf{K}_{2}}^{p}\right]$ is required for exponential convergence for the proposed control law in this paper. In other words, unless otherwise noted, $\left[\mathbf{L}_{\mathbf{K}_{1},-}^{p} \mathbf{K}_{2}\right]$ is assumed to have no zero eigenvalue. 
We are well poised to introduce the main theorems of the present paper. First, the following condition should be true for exponential convergence to the common desired trajectory $\mathbf{q}_{d}$.

Theorem III.1 Global Exponential Convergence to the Desired Trajectory

If $\left[\mathbf{L}_{\mathbf{K}_{1},-\mathbf{K}_{2}}^{p}\right]$ is positive definite, then every member of the network follows the desired trajectory $\mathbf{q}_{d}$ exponentially fast regardless of initial conditions.

$$
\left[\mathbf{L}_{\mathbf{K}_{1},-\mathbf{K}_{2}}^{p}\right]>0
$$

In other words, if $\mathbf{K}_{1}-2 \mathbf{K}_{2}>0$, then $\mathbf{q}_{i},(i=1,2, \cdots, p, p \geq 3)$ converges to $\mathbf{q}_{d}$ exponentially fast from any initial conditions. For two-spacecraft systems $(p=2), \mathbf{K}_{1}-\mathbf{K}_{2}>0$ needs to be true instead.

Proof We can cancel out the $\left[\mathbf{U}_{\mathbf{K}_{2}}^{p}\right]$ matrix term in (21) to obtain

$$
[\mathbf{M}] \dot{\mathbf{x}}+[\mathbf{C}] \mathbf{x}+\left[\mathbf{L}_{\mathbf{K}_{1},-\mathbf{K}_{2}}^{p}\right] \mathbf{x}=\mathbf{0} .
$$

Equation (23) corresponds to a conventional tracking problem. We use contraction theory (see the Appendix) to prove that $\mathbf{x}$ tends to zero exponentially with $\left[\mathbf{L}_{\mathbf{K}_{1},-\mathbf{K}_{2}}^{p}\right]>0$. For example, consider the virtual system of $\mathbf{y}$ obtained by replacing $\mathbf{x}$ with $\mathbf{y}$ in (23).

$$
[\mathbf{M}] \dot{\mathbf{y}}+[\mathbf{C}] \mathbf{y}+\left[\mathbf{L}_{\mathbf{K}_{1},-\mathbf{K}_{2}}^{p}\right] \mathbf{y}=\mathbf{0}
$$

This virtual $\mathbf{y}$ system has two particular solutions: $\mathbf{x}=\left(\mathbf{s}_{1}, \cdots, \mathbf{s}_{p}\right)^{T}$ and $\mathbf{0}$. The squared-length analysis with respect to the positive-definite metric $[\mathbf{M}]$ yields

$$
\begin{aligned}
\frac{d}{d t}\left(\delta \mathbf{y}^{T}[\mathbf{M}] \delta \mathbf{y}\right) & =2 \delta \mathbf{y}^{T}[\mathbf{M}] \delta \dot{\mathbf{y}}+\delta \mathbf{y}^{T}[\dot{\mathbf{M}}] \delta \mathbf{y} \\
& =-2 \delta \mathbf{y}^{T}\left([\mathbf{C}] \delta \mathbf{y}+\left[\mathbf{L}_{\mathbf{K}_{1},-\mathbf{K}_{2}}^{p}\right] \delta \mathbf{y}\right)+\delta \mathbf{y}^{T}[\dot{\mathbf{M}}] \delta \mathbf{y}=-2 \delta \mathbf{y}^{T}\left[\mathbf{L}_{\mathbf{K}_{1},-\mathbf{K}_{2}}^{p}\right] \delta \mathbf{y}
\end{aligned}
$$

where we used the skew-symmetric property of $[\dot{\mathbf{M}}]-2[\mathbf{C}]$.

Accordingly, $\left[\mathbf{L}_{\mathbf{K}_{1},-\mathbf{K}_{2}}^{p}\right]>0$ will make the system contracting, thus all solutions of $\mathbf{y}$ converge to a single trajectory exponentially fast. This in turn indicates that the composite variable of each robot tends to zero exponentially $(\mathbf{s} \rightarrow \mathbf{0})$. By the definition of $\mathbf{s}_{i}=\dot{\mathbf{q}}-\dot{\mathbf{q}}_{d}+\boldsymbol{\Lambda}\left(\mathbf{q}_{i}-\mathbf{q}_{d}\right)$, the exponential convergence of $\mathbf{q}_{i}$ to the common reference trajectory $\mathbf{q}_{d}$ is proven (see the hierarchical combination in Theorem VII.2). The positive-definiteness of $\left[\mathbf{L}_{\mathbf{K}_{1},-\mathbf{K}_{2}}^{p}\right]$ corresponds to $\mathbf{K}_{1}-\mathbf{K}_{2}>0$ for two-spacecraft systems $(p=2)$. For a network consisting of more than two spacecraft $(p \geq 3)$, it can be shown that $\mathbf{K}_{1}-2 \mathbf{K}_{2}$ is a sufficient condition of the positive-definiteness of $\left[\mathbf{L}_{\mathbf{K}_{1},-\mathbf{K}_{2}}^{p}\right]$ given $\mathbf{K}_{1}>0, \mathbf{K}_{2}>0$.

The next question to be addressed is how to guarantee the synchronization of the individual dynamics.

Theorem III.2 Synchronization of Multiple Identical or Heterogeneous Spacecraft

Suppose the conditions in Theorem III.1 are true, thus the individual dynamics are exponentially tracking the common desired trajectory. A swarm of $p$ spacecraft synchronize exponentially from any initial conditions if $\exists$ diagonal matrices $\mathbf{K}_{1}>0, \mathbf{K}_{2}>0$ such that

$$
\left[\mathbf{L}_{\mathbf{K}_{1},-\mathbf{K}_{2}}^{p}\right]+\left[\mathbf{U}_{\mathbf{K}_{2}}^{p}\right]>0
$$

In addition, $\boldsymbol{\Lambda}$ is a positive diagonal matrix defining a stable composite variable $\mathbf{s}_{i}=\dot{\widetilde{\mathbf{q}}}_{i}+\boldsymbol{\Lambda} \widetilde{\mathbf{q}}_{i}$.

This theorem corresponds to synchronization with stable tracking. The proof is expanded in Section IV by separating the two different time scales of the closed-loop dynamics. Multiple dynamics need not be identical to achieve stable synchronization.

It is useful to note that the above condition corresponds to $\mathbf{K}_{1}+\mathbf{K}_{2}>0$ for two-spacecraft and threespacecraft networks $(p=2,3)$. A four-robot network $(p=4)$ would require $\mathbf{K}_{1}+2 \mathbf{K}_{2}>0$.

Note that we can render the system synchronized first, then follow the common trajectory by tuning the gains properly. For an example of a two-spacecraft network, $\mathbf{K}_{2}>0$ ensures that the two spacecraft synchronize faster than they follow the common desired trajectory, since $\mathbf{K}_{1}+\mathbf{K}_{2}>\mathbf{K}_{1}-\mathbf{K}_{2}$ for $\forall \mathbf{K}_{2}>0$. This indicates that there exist two different time-scales in the closed-loop systems constructed with the proposed controllers. For two-spacecraft systems, the convergence of exponential tracking is proportional to $\mathbf{K}_{1}-\mathbf{K}_{2}$ whereas the synchronization has a convergence rate of $\mathbf{K}_{1}+\mathbf{K}_{2}$. This multi-time-scale behavior will be exploited in the subsequent sections. 


\section{Proof of Nonlinear Stability for Exponential Synchronization}

We prove Theorem III.2 for the exponential synchronization of multiple nonlinear dynamics in this section. First, we describe the difficulties inherent in proving the synchronization of highly nonlinear systems in Section A. We then focus on the two-spacecraft synchronization problem in Section B. The key result can generalized for an arbitrary number of spacecraft.

\section{A. Difficulties with the Synchronization of Lagrangian Systems}

The difficulties associated with nonlinear time-varying inertia matrices can be easily demonstrated with the following two-spacecraft example. The closed-loop dynamics of two identical spacecraft from (21) becomes

$$
\begin{array}{r}
\mathbf{M}\left(\mathbf{q}_{1}\right) \dot{\mathbf{s}}_{1}+\mathbf{C}\left(\mathbf{q}_{1}, \dot{\mathbf{q}}_{1}\right) \mathbf{s}_{1}+\left(\mathbf{K}_{1}+\mathbf{K}_{2}\right) \mathbf{s}_{1}=\mathbf{u}(t) \\
\mathbf{M}\left(\mathbf{q}_{2}\right) \dot{\mathbf{s}}_{2}+\mathbf{C}\left(\mathbf{q}_{2}, \dot{\mathbf{q}}_{2}\right) \mathbf{s}_{2}+\left(\mathbf{K}_{1}+\mathbf{K}_{2}\right) \mathbf{s}_{2}=\mathbf{u}(t) \\
\mathbf{u}(t)=\mathbf{K}_{2}\left(\mathbf{s}_{1}+\mathbf{s}_{2}\right)
\end{array}
$$

Direct application of synchronization (Theorem VII.4) appears elusive because the original dynamics in (2) with the control law (18) is in general not contracting. Since (2) is a second-order differential equation, this can also be viewed as a high-order contraction problem. ${ }^{15}$ If we transform the dynamics into a firstorder canonical form, we have to prove that they are contracting in the same metric while preserving the input symmetry. ${ }^{26}$ For example, multiplying (26) by $\mathbf{M}^{-1}$ breaks the input symmetry: i.e., $\mathbf{M}^{-1}\left(\mathbf{q}_{1}\right) \mathbf{u}(t) \neq$ $\mathbf{M}^{-1}\left(\mathbf{q}_{2}\right) \mathbf{u}(t)$. In essence, $\mathbf{M}\left(\mathbf{q}_{1}\right) \neq \mathbf{M}\left(\mathbf{q}_{2}\right)$ makes this problem intractable in general.

Instead, suppose that $\mathbf{M}(\mathbf{q})$ remains constant, thereby making $\mathbf{C}(\mathbf{q}, \dot{\mathbf{q}})$ zero. Then, we can easily prove $\mathbf{s}_{1}$ and $\mathbf{s}_{2}$ tend to each other from

$$
\begin{aligned}
& \mathbf{M} \dot{\mathbf{s}}_{1}+\left(\mathbf{K}_{1}+\mathbf{K}_{2}\right) \mathbf{s}_{1}=\mathbf{K}_{2}\left(\mathbf{s}_{1}+\mathbf{s}_{2}\right) \\
& \mathbf{M} \dot{\mathbf{s}}_{2}+\left(\mathbf{K}_{1}+\mathbf{K}_{2}\right) \mathbf{s}_{2}=\mathbf{K}_{2}\left(\mathbf{s}_{1}+\mathbf{s}_{2}\right)
\end{aligned}
$$

Since the virtual system with the common input $\mathbf{u}(t)=\mathbf{K}_{2}\left(\mathbf{s}_{1}+\mathbf{s}_{2}\right)$

$$
\mathbf{M} \dot{\mathbf{y}}+\left(\mathbf{K}_{1}+\mathbf{K}_{2}\right) \mathbf{y}=\mathbf{u}(t)
$$

is contracting with $\mathbf{K}_{1}+\mathbf{K}_{2}>0$. Hence, its particular solutions $\mathbf{s}_{1}$ and $\mathbf{s}_{2}$ tend to each other exponentially fast according to the synchronization theorem (Theorem VII.4). Without loss of generality, this result can easily be extended to arbitrarily large networks. The synchronization of a large network with a constant metric is already discussed in Ref. 37 using contraction analysis.

We now turn to a much more difficult problem focused on the synchronization of two spacecraft with non-constant nonlinear metrics $\left(\mathbf{M}\left(\mathbf{q}_{1}\right) \neq \mathbf{M}\left(\mathbf{q}_{2}\right)\right)$.

\section{B. Contraction with Two Time-Scales}

As mentioned earlier, for two-spacecraft systems, the convergence rate of exponential tracking to the desired trajectory is proportional to $\mathbf{K}_{1}-\mathbf{K}_{2}$ whereas the synchronization counterpart has the faster convergence of $\mathbf{K}_{1}+\mathbf{K}_{2}$.

$$
\mathbf{K}_{1}+\mathbf{K}_{2}>\mathbf{K}_{1}-\mathbf{K}_{2}, \forall \mathbf{K}_{2}>0
$$

This multi timescale behavior is graphically illustrated in Figure 3. The figure ${ }^{\mathrm{a}}$ depicts that $\mathbf{s}_{1}$ and $\mathbf{s}_{2}$ synchronize first, then they converge to the desired trajectory while staying together. This observation motivates separation of the two different time scales, namely $\mathbf{K}_{1}+\mathbf{K}_{2}$ and $\mathbf{K}_{1}-\mathbf{K}_{2}$.

Recall the closed-loop dynamics given in (21):

$$
[\mathbf{M}] \dot{\mathbf{x}}+[\mathbf{C}] \mathbf{x}+\left[\mathbf{L}_{\mathbf{K}_{1},-\mathbf{K}_{2}}^{p}\right] \mathbf{x}=\mathbf{0}
$$

Since $\left[\mathbf{L}_{\mathbf{K}_{1},-\mathbf{K}_{2}}^{p}\right]$ is a real symmetric matrix, we can perform the spectral decomposition, as in spectral graph theory, ${ }^{3}$

$$
\begin{aligned}
& {\left[\mathbf{L}_{\mathbf{K}_{1},-\mathbf{K}_{2}}^{p}\right]=\mathbf{V}[\mathbf{D}] \mathbf{V}^{T}} \\
& \mathbf{V}^{T}\left[\mathbf{L}_{\mathbf{K}_{1},-\mathbf{K}_{2}}^{p}\right] \mathbf{V}=[\mathbf{D}]
\end{aligned}
$$

a The picture is slightly exaggerated because $\mathbf{s}_{1}$ and $\mathbf{s}_{2}$ appear overlapped when they synchronize. Strictly speaking, their difference is decreasing exponentially to zero but they will never be the same unless time tends to infinity. 


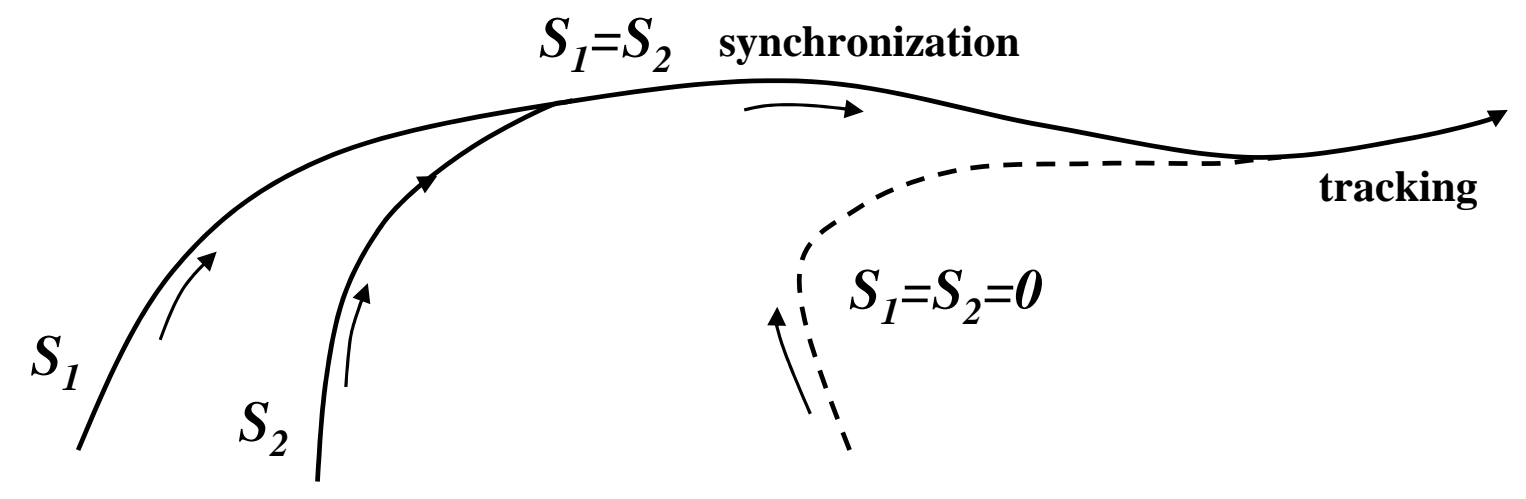

Figure 3. Multiple timescales of synchronization (faster) and tracking (slower). The dashed line indicates the desired trajectory. Arrows indicate increasing time. ${ }^{6}$

where $[\mathbf{D}]$ is a block diagonal matrix and $\mathbf{V}^{T} \mathbf{V}=\mathbf{V V}^{T}=\mathbf{I}$. Note that symmetry of a matrix gives rise to real eigenvalues and perpendicular eigenvectors. ${ }^{32}$

Pre-multiplying (30) by $\mathbf{V}^{T}$ and setting $\mathbf{x}=\mathbf{V V}^{T} \mathbf{x}$ result in

$$
\left(\mathbf{V}^{T}[\mathbf{M}] \mathbf{V}\right) \mathbf{V}^{T} \dot{\mathbf{x}}+\left(\mathbf{V}^{T}[\mathbf{C}] \mathbf{V}\right) \mathbf{V}^{T} \mathbf{x}+\left(\mathbf{V}^{T}\left[\mathbf{L}_{\mathbf{K}_{1},-\mathbf{K}_{2}}^{p}\right] \mathbf{V}\right) \mathbf{V}^{T} \mathbf{x}=\mathbf{0}
$$

By setting $\mathbf{V}^{T} \mathbf{x}=\mathbf{z},(32)$ becomes

$$
\left(\mathbf{V}^{T}[\mathbf{M}] \mathbf{V}\right) \dot{\mathbf{z}}+\left(\mathbf{V}^{T}[\mathbf{C}] \mathbf{V}\right) \mathbf{z}+[\mathbf{D}] \mathbf{z}=\mathbf{0}
$$

Then, we can develop the squared-length analysis similar to the previous section. Notice that $\left(\mathbf{V}^{T}[\mathbf{M}] \mathbf{V}\right)$ is always symmetric positive definite since $[\mathbf{M}]$ is symmetric positive definite. ${ }^{32}$ For the case of a twospacecraft network, we can easily verify that (33) becomes

$$
\left[\mathbf{M}_{2 T}\right] \dot{\mathbf{z}}+\left[\mathbf{C}_{2 T}\right] \mathbf{z}+\left[\mathbf{D}_{2}\right] \mathbf{z}=\mathbf{0}
$$

where

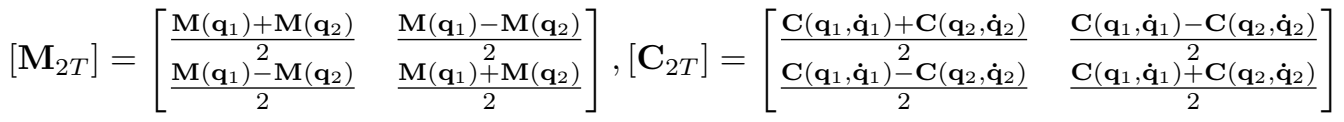

$$
\begin{aligned}
& {\left[\mathbf{D}_{2}\right]=\left[\begin{array}{cc}
\mathbf{K}_{1}-\mathbf{K}_{2} & \mathbf{0} \\
\mathbf{0} & \mathbf{K}_{1}+\mathbf{K}_{2}
\end{array}\right], \quad \mathbf{V}=\left[\begin{array}{cc}
\frac{1}{\sqrt{2}} \mathbf{I} & \frac{1}{\sqrt{2}} \mathbf{I} \\
\frac{1}{\sqrt{2}} \mathbf{I} & -\frac{1}{\sqrt{2}} \mathbf{I}
\end{array}\right]}
\end{aligned}
$$

Consider the virtual system of $\mathbf{y}_{1}$ and $\mathbf{y}_{2}$

$$
\left[\mathbf{M}_{2 T}\right] \dot{\mathbf{y}}+\left[\mathbf{C}_{2 T}\right] \mathbf{y}+\left[\mathbf{D}_{2}\right] \mathbf{y}=\mathbf{0}
$$

that has the following two particular solutions:

$$
\left(\mathbf{y}_{1}=\frac{\mathbf{s}_{1}+\mathbf{s}_{2}}{\sqrt{2}}, \mathbf{y}_{2}=\frac{\mathbf{s}_{1}-\mathbf{s}_{2}}{\sqrt{2}}\right)^{T} \text { and } \quad\left(\mathbf{y}_{1}=\mathbf{0}, \mathbf{y}_{2}=\mathbf{0}\right)^{T}
$$

For $\mathbf{K}_{2}>0$ and $\mathbf{K}_{1}-\mathbf{K}_{2}>0$, which also lead to $\mathbf{K}_{1}+\mathbf{K}_{2}>\mathbf{K}_{1}-\mathbf{K}_{2}$, we can show that the above virtual system is contracting. We take the symmetric block matrix $\left[\mathbf{M}_{2 T}\right]$ as our contraction metric.

Performing the squared-length analysis with respect to this metric yields

$$
\begin{aligned}
\frac{d}{d t}\left(\delta \mathbf{y}^{T}\left[\mathbf{M}_{2 T}\right] \delta \mathbf{y}\right) & =2 \delta \mathbf{y}^{T}\left[\mathbf{M}_{2 T}\right] \delta \dot{\mathbf{y}}+\delta \mathbf{y}^{T}\left[\dot{\mathbf{M}}_{2 T}\right] \delta \mathbf{y} \\
& =-2 \delta \mathbf{y}^{T}\left(\left[\mathbf{C}_{2 T}\right] \delta \mathbf{y}+\left[\mathbf{D}_{2}\right] \delta \mathbf{y}\right)+\delta \mathbf{y}^{T}\left[\dot{\mathbf{M}}_{2 T}\right] \delta \mathbf{y} \\
& =-2 \delta \mathbf{y}^{T}\left[\mathbf{D}_{2}\right] \delta \mathbf{y}
\end{aligned}
$$


where we used the skew-symmetric property of $\left[\dot{\mathbf{M}}_{2 T}\right]-2\left[\mathbf{C}_{2 T}\right]$.

We can recall the stability analysis of the trajectory tracking, depending on the sign of $\mathbf{K}_{1}-\mathbf{K}_{2}$ (see Theorem III.1). In the following, three possible cases are classified by the sign of $\mathbf{K}_{1}-\mathbf{K}_{2}$, and discussed respectively. Namely, stable tracking with $\mathbf{K}_{1}-\mathbf{K}_{2}>0$; indifferent tracking with $\mathbf{K}_{1}-\mathbf{K}_{2}=\mathbf{0}$; unstable tracking with $\mathbf{K}_{1}-\mathbf{K}_{2}<0$.

Case I: $\mathbf{K}_{1}-\mathbf{K}_{2}>0$

If $\mathbf{K}_{1}+\mathbf{K}_{2}>\mathbf{K}_{1}-\mathbf{K}_{2}>0$, the rate of the virtual length in (38) is uniformly negative definite for nonzero $\delta \mathbf{y}_{1}$ and $\delta \mathbf{y}_{2}$ :

$$
\frac{d}{d t}\left(\delta \mathbf{y}^{T}\left[\mathbf{M}_{2 T}\right] \delta \mathbf{y}\right)-2 \delta \mathbf{y}^{T}\left[\mathbf{D}_{2}\right] \delta \mathbf{y}<0
$$

Consequently, the combined virtual system in (36) is contracting. In other words, $\delta \mathbf{y}_{1}, \delta \mathbf{y}_{2} \rightarrow \mathbf{0}$ exponentially fast. This in turn implies all solutions of $\mathbf{y}_{1}$ and $\mathbf{y}_{2}$ tend to the single trajectory. As a result, $\mathbf{s}_{1}+\mathbf{s}_{2}$ and $\mathbf{s}_{1}-\mathbf{s}_{2}$ tend to zero exponentially. It is straightforward to show that $\mathbf{s}_{1} \rightarrow \mathbf{s}_{2}$ also hierarchically makes $\mathbf{q}_{1}$ tend to $\mathbf{q}_{2}$ exponentially (see Theorem VII.2).

From the definition of the composite variables in (19), we can find the following contracting dynamics,

$$
\left(\dot{\mathbf{q}}_{1}-\dot{\mathbf{q}}_{2}\right)+\boldsymbol{\Lambda}\left(\mathbf{q}_{1}-\mathbf{q}_{2}\right)=\mathbf{s}_{1}-\mathbf{s}_{2}
$$

Note that $\dot{\mathbf{y}}+\mathbf{\Lambda} \mathbf{y}=0$ is contracting with $\boldsymbol{\Lambda}>0$. Consequently, $\boldsymbol{\Lambda}>0$ and $\mathbf{s}_{1} \rightarrow \mathbf{s}_{2}$ make $\mathbf{q}_{1} \rightarrow \mathbf{q}_{2}$ exponentially fast. In the case of two identical spacecraft, this also implies that the diagonal terms of the metric, $\frac{\mathbf{M}\left(\mathbf{q}_{1}\right)-\mathbf{M}\left(\mathbf{q}_{2}\right)}{2}$ tend to zero exponentially, thereby eliminating the coupling of the inertia term.

$$
\left[\mathbf{M}_{2 T}\right]=\left[\begin{array}{ll}
\frac{\mathbf{M}\left(\mathbf{q}_{1}\right)+\mathbf{M}\left(\mathbf{q}_{2}\right)}{2} & \frac{\mathbf{M}\left(\mathbf{q}_{1}\right)-\mathbf{M}\left(\mathbf{q}_{2}\right)}{2} \\
\frac{\mathbf{M}\left(\mathbf{q}_{1}\right)-\mathbf{M}\left(\mathbf{q}_{2}\right)}{2} & \frac{\mathbf{M}\left(\mathbf{q}_{1}\right)+\mathbf{M}\left(\mathbf{q}_{2}\right)}{2}
\end{array}\right] \longrightarrow\left[\begin{array}{cc}
\mathbf{M}\left(\mathbf{q}_{1}\right) & \mathbf{0} \\
\mathbf{0} & \mathbf{M}\left(\mathbf{q}_{1}\right)
\end{array}\right]
$$

Once the inertia matrix is sufficiently close to the diagonal matrix in (41), the squared-length analysis in (38) reduces to

$$
\frac{d}{d t} \delta \mathbf{y}_{1}^{T} \mathbf{M}\left(\mathbf{q}_{1}\right) \delta \mathbf{y}_{1}=-2 \delta \mathbf{y}_{1}^{T}\left[\mathbf{K}_{1}-\mathbf{K}_{2}\right] \delta \mathbf{y}_{1}, \quad \text { and } \frac{d}{d t} \delta \mathbf{y}_{2}{ }^{T} \mathbf{M}\left(\mathbf{q}_{1}\right) \delta \mathbf{y}_{2}=-2 \delta \mathbf{y}_{2}{ }^{T}\left[\mathbf{K}_{1}+\mathbf{K}_{2}\right] \delta \mathbf{y}_{2}
$$

This in turn implies that the convergence rate of tracking, $\left(\delta \mathbf{y}_{1}\right)$ is proportional to $\mathbf{K}_{1}-\mathbf{K}_{2}$ while the synchronization, $\left(\delta \mathbf{y}_{2}\right)$ occurs at a faster convergence rate, $\mathbf{K}_{1}+\mathbf{K}_{2}$. This completes the proof of Theorem III.2.

Case II: $\mathbf{K}_{1}-\mathbf{K}_{2}=\mathbf{0}$

We can also consider a case with $\mathbf{K}_{1}-\mathbf{K}_{2}=\mathbf{0}$, which fails the exponential stability condition in Theorem III.1. The combined virtual system per se is then semi-contracting (asymptotically stable) ${ }^{14}$ since the squared-length analysis in (38) yields the negative semi-definite matrix:

$$
\frac{d}{d t}\left(\delta \mathbf{y}^{T}\left[\mathbf{M}_{2 T}\right] \delta \mathbf{y}\right)=\left(\begin{array}{c}
\delta \mathbf{y}_{1} \\
\delta \mathbf{y}_{2}
\end{array}\right)^{T}\left[\begin{array}{cc}
\mathbf{0} & \mathbf{0} \\
\mathbf{0} & -2\left(\mathbf{K}_{1}+\mathbf{K}_{2}\right)
\end{array}\right]\left(\begin{array}{l}
\delta \mathbf{y}_{1} \\
\delta \mathbf{y}_{2}
\end{array}\right) \leq 0
$$

While $\delta \mathbf{y}_{1}$, representing the tracking dynamics, remains in a finite ball due to $\mathbf{K}_{1}-\mathbf{K}_{2}=0, \delta \mathbf{y}_{2}$ tends to zero exponentially due to $-2\left(\mathbf{K}_{1}+\mathbf{K}_{2}\right)<0$. This result can be proven as follows. $\dot{V}$ is uniformly continuous since a bounded $\delta \dot{\mathbf{y}}_{2}$ from (36) leads to a bounded $\ddot{V}$ from

$$
\ddot{V}=-4 \delta \mathbf{y}_{2}^{T}\left(\mathbf{K}_{1}+\mathbf{K}_{2}\right) \delta \dot{\mathbf{y}}_{2}
$$

Due to $\dot{V} \leq 0$, the use of Barbalat's lemma ${ }^{18}$ verifies that $\dot{V} \rightarrow 0$ as $t \rightarrow \infty$. This implies that $\delta \mathbf{y}_{2}$ tends to zero asymptotically fast.

We introduce the third virtual variable $\mathbf{y}_{3}$ to verify a hierarchical combination of $\mathbf{s}_{1}-\mathbf{s}_{2}$ and $\mathbf{q}_{1}-\mathbf{q}_{2}$, stated in (40):

$$
\delta \dot{\mathbf{y}}_{3}+\mathbf{\Lambda} \delta \mathbf{y}_{3}=\delta \mathbf{y}_{2}
$$


where $\mathbf{y}_{3}$ has two particular solutions: $\mathbf{y}_{3}=\mathbf{q}_{1}-\mathbf{q}_{2}$ when $\mathbf{y}_{2}=\mathbf{s}_{1}-\mathbf{s}_{2}$, and $\mathbf{y}_{3}=\mathbf{0}$ when $\mathbf{y}_{2}=\mathbf{0}$.

Since $\delta \mathbf{y}_{2} \rightarrow \mathbf{0}$ asymptotically by Barbalat's lemma, $\delta \mathbf{y}_{3}$ tends to zero with a positive $\boldsymbol{\Lambda}>0$. Consequently, $\delta \mathbf{y}_{3} \rightarrow \mathbf{0}$ indicates $\mathbf{q}_{1} \rightarrow \mathbf{q}_{2}$. This will eventually decouple the metric matrix with $\boldsymbol{\Lambda}>0$, as seen in (41), since $\mathbf{M}\left(\mathbf{q}_{1}\right)-\mathbf{M}\left(\mathbf{q}_{2}\right)$ tends to zero simultaneously as $\mathbf{q}_{1} \rightarrow \mathbf{q}_{2}$. As a result, when $\mathbf{M}\left(\mathbf{q}_{1}\right)-\mathbf{M}\left(\mathbf{q}_{2}\right)$ is sufficiently close to zero, the convergence of $\delta \mathbf{y}_{2} \rightarrow 0$ turns exponential.

We can conclude that $\mathbf{q}_{1}$ and $\mathbf{q}_{2}$ synchronize even with $\mathbf{K}_{1}-\mathbf{K}_{2}=\mathbf{0}$. Furthermore, we can prove synchronization in the presence of tracking instability $\left(\mathbf{K}_{1}-\mathbf{K}_{2}<0\right)$ with sufficiently small $\left\|\mathbf{K}_{1}-\mathbf{K}_{2}\right\|$ by decoupling the unstable dynamics from the stable synchronization dynamics as follows.

Case III: $\mathbf{K}_{1}-\mathbf{K}_{2}<0$

Consider a case when the individual tracking system is unstable with $\mathbf{K}_{1}-\mathbf{K}_{2}<0$. This case warrants further discussion. In essence, we show in Ref. 6 that the synchronization can occur fast enough to overcome the tracking instability. In this case, $\boldsymbol{\Lambda}$ should be sufficiently large such that $\|\boldsymbol{\Lambda}\| \gg \frac{\left\|\mathbf{K}_{1}-\mathbf{K}_{2}\right\|}{\underline{\sigma}(\mathbf{M}(\mathbf{q}))}$ for $p=2$ or $\|\boldsymbol{\Lambda}\| \gg \frac{\left\|\mathbf{K}_{1}-2 \mathbf{K}_{2}\right\|}{\underline{\sigma}(\mathbf{M}(\mathbf{q}))}$ for $p \geq 3$, where $\underline{\sigma}(\cdot)$ denotes the smallest singular value. In contrast with Theorem III.2, the individual dynamics must be identical in the unstable tracking case. We refer the readers to Ref. 6,7 for the detailed proof.

We can extend the method in this section to arbitrarily large networks. For example, a network of three spacecraft has the following $\mathbf{V}$ whose columns are orthonormal eigenvectors of $\left[\mathbf{L}_{\mathbf{K}_{1},-\mathbf{K}_{2}}^{p=3}\right]$ :

$$
\mathbf{V}=\left[\begin{array}{ccc}
-\frac{1}{\sqrt{3}} \mathbf{I} & -\frac{2}{\sqrt{6}} \mathbf{I} & \mathbf{0} \\
-\frac{1}{\sqrt{3}} \mathbf{I} & \frac{1}{\sqrt{6}} \mathbf{I} & -\frac{1}{\sqrt{2}} \mathbf{I} \\
-\frac{1}{\sqrt{3}} \mathbf{I} & \frac{1}{\sqrt{6}} \mathbf{I} & \frac{1}{\sqrt{2}} \mathbf{I}
\end{array}\right]
$$

Note that $\mathbf{V}$ is a unitary matrix such that $\mathbf{V}^{T} \mathbf{V}=\mathbf{V} \mathbf{V}^{T}=\mathbf{I}$.

The new transformed inertia matrix, $\mathbf{V}^{T}[\mathbf{M}] \mathbf{V}$ is written as

$$
\left[\begin{array}{ccc}
\frac{1}{3} \mathbf{M}_{1}+\frac{1}{3} \mathbf{M}_{2}+\frac{1}{3} \mathbf{M}_{3} & \frac{\sqrt{2}}{3} \mathbf{M}_{1}-\frac{1}{3 \sqrt{2}} \mathbf{M}_{2}-\frac{1}{3 \sqrt{2}} \mathbf{M}_{3} & \frac{1}{\sqrt{6}} \mathbf{M}_{2}-\frac{1}{\sqrt{6}} \mathbf{M}_{3} \\
\frac{\sqrt{2}}{3} \mathbf{M}_{1}-\frac{1}{3 \sqrt{2}} \mathbf{M}_{2}-\frac{1}{3 \sqrt{2}} \mathbf{M}_{3} & \frac{2}{3} \mathbf{M}_{1}+\frac{1}{6} \mathbf{M}_{2}+\frac{1}{6} \mathbf{M}_{3} & -\frac{1}{2 \sqrt{3}} \mathbf{M}_{2}+\frac{1}{2 \sqrt{3}} \mathbf{M}_{3} \\
\frac{1}{\sqrt{6}} \mathbf{M}_{2}-\frac{1}{\sqrt{6}} \mathbf{M}_{3} & -\frac{1}{2 \sqrt{3}} \mathbf{M}_{2}+\frac{1}{2 \sqrt{3}} \mathbf{M}_{3} & \frac{1}{2} \mathbf{M}_{2}+\frac{1}{2} \mathbf{M}_{3}
\end{array}\right]
$$

where $\mathbf{M}_{1}=\mathbf{M}\left(\mathbf{q}_{1}\right), \mathbf{M}_{2}=\mathbf{M}\left(\mathbf{q}_{2}\right)$, and $\mathbf{M}_{3}=\mathbf{M}\left(\mathbf{q}_{3}\right)$ for notational simplicity. This matrix is symmetric positive definite. Also notice that its off-diagonal terms vanish as $\mathbf{q}_{1} \rightarrow \mathbf{q}_{2}, \mathbf{q}_{2} \rightarrow \mathbf{q}_{3}$ :

$$
\mathbf{V}^{T}[\mathbf{M}] \mathbf{V} \longrightarrow\left[\begin{array}{ccc}
\mathbf{M}(\mathbf{q}) & \mathbf{0} & \mathbf{0} \\
\mathbf{0} & \mathbf{M}(\mathbf{q}) & \mathbf{0} \\
\mathbf{0} & \mathbf{0} & \mathbf{M}(\mathbf{q})
\end{array}\right]
$$

where $\mathbf{q}=\mathbf{q}_{1}=\mathbf{q}_{2}=\mathbf{q}_{3}$.

The diagonal matrix $[\mathbf{D}]$ is also computed as

$$
\left[\begin{array}{ccc}
\mathbf{K}_{1}-2 \mathbf{K}_{2} & \mathbf{0} & \mathbf{0} \\
\mathbf{0} & \mathbf{K}_{1}+\mathbf{K}_{2} & \mathbf{0} \\
\mathbf{0} & \mathbf{0} & \mathbf{K}_{1}+\mathbf{K}_{2}
\end{array}\right]
$$

\section{Extensions and Examples}

Let us examine the effectiveness of the proposed control law in a few examples. 


\section{A. Synchronization with Partial Degrees-of-Freedom Coupling}

In this section, we consider multiple spacecraft with partially coupled variables. For instance, we can assume that only the first and third MRP variables $\left(q_{1}\right.$ and $\left.q_{3}\right)$ are coupled in a two-spacecraft system such that

$$
\begin{aligned}
& \tau_{1}=\mathbf{M}\left(\mathbf{q}_{1}\right) \ddot{\mathbf{q}}_{1 r}+\mathbf{C}\left(\mathbf{q}_{1}, \dot{\mathbf{q}}_{1}\right) \dot{\mathbf{q}}_{1 r}+\mathbf{g}\left(\mathbf{q}_{1}\right)-\mathbf{K}_{1} \mathbf{s}_{1}+\mathbf{K}_{2}\left(\begin{array}{lll}
\dot{\widetilde{q}}_{1} & 0 & \dot{\widetilde{q}}_{3}
\end{array}\right)_{\mathbf{q}_{2}}^{T}+\mathbf{K}_{2} \boldsymbol{\Lambda}\left(\begin{array}{ccc}
\widetilde{q}_{1} & 0 & \widetilde{q}_{3}
\end{array}\right)_{\mathbf{q}_{2}}^{T} \\
& \tau_{2}=\mathbf{M}\left(\mathbf{q}_{2}\right) \ddot{\mathbf{q}}_{2 r}+\mathbf{C}\left(\mathbf{q}_{2}, \dot{\mathbf{q}}_{2}\right) \dot{\mathbf{q}}_{2 r}+\mathbf{g}\left(\mathbf{q}_{2}\right)-\mathbf{K}_{1} \mathbf{s}_{2}+\mathbf{K}_{2}\left(\begin{array}{lll}
\dot{\widetilde{q}}_{1} & 0 & \dot{\widetilde{q}}_{3}
\end{array}\right)_{\mathbf{q}_{1}}^{T}+\mathbf{K}_{2} \boldsymbol{\Lambda}\left(\begin{array}{lll}
\widetilde{q}_{1} & 0 & \widetilde{q}_{3}
\end{array}\right)_{\mathbf{q}_{1}}^{T}
\end{aligned}
$$

Nevertheless, Theorems III.1 and III.2 are true with diagonal matrices, $\mathbf{K}_{1}, \mathbf{K}_{2}$ and $\boldsymbol{\Lambda}$, which can be verified by writing the closed-loop system as in (26):

$$
\begin{array}{r}
\mathbf{M}\left(\mathbf{q}_{1}\right) \dot{\mathbf{s}}_{1}+\mathbf{C}\left(\mathbf{q}_{1}, \dot{\mathbf{q}}_{1}\right) \mathbf{s}_{1}+\left(\mathbf{K}_{1}+\mathbf{K}_{2} \mathbf{P}\right) \mathbf{s}_{1}=\mathbf{u}(t) \\
\mathbf{M}\left(\mathbf{q}_{2}\right) \dot{\mathbf{s}}_{2}+\mathbf{C}\left(\mathbf{q}_{2}, \dot{\mathbf{q}}_{2}\right) \mathbf{s}_{2}+\left(\mathbf{K}_{1}+\mathbf{K}_{2} \mathbf{P}\right) \mathbf{s}_{2}=\mathbf{u}(t) \\
\mathbf{u}(t)=\mathbf{K}_{2} \mathbf{P}\left(\mathbf{s}_{1}+\mathbf{s}_{2}\right)
\end{array}
$$

where $\mathbf{P}=\operatorname{diag}(1,0,1)$.

It is straightforward to prove that Theorems III.1 and III.2 still hold. This is because

$$
\left(\mathbf{K}_{1}+\mathbf{K}_{2} \mathbf{P}\right) \text { and }\left(\mathbf{K}_{1}-\mathbf{K}_{2} \mathbf{P}\right)
$$

are still uniformly positive definite, enabling exponential synchronization and exponential convergence to the desired trajectory, respectively. Hence, we did not break any assumptions in the proof of Theorem III.2.

\section{B. Attitude Synchronization of Two Spacecraft}

The proposed control law in (18) is simulated for two identical spacecraft, as shown in Figure 4(a). The spacecraft inertial matrix is $\mathbf{J}_{\mathbf{s} / \mathbf{c}}=\left[\begin{array}{ccc}150 & 0 & -100 \\ 0 & 270 & 0 \\ -100 & 0 & 300\end{array}\right]\left[\mathrm{kgm}^{2}\right]$. The control gains are defined as $\mathbf{K}_{1}=$ $300 \mathbf{I}, \mathbf{K}_{2}=100 \mathbf{I}$, and $\boldsymbol{\Lambda}=20 \mathbf{I}$. The reference trajectories are defined as $\mathbf{q}_{1 d}=0.3 \sin (2 \pi(0.01) t), \mathbf{q}_{2 d}=$ $0.2 \sin (2 \pi(0.02) t+\pi / 6)$, and $\mathbf{q}_{3 d}=0$. The first spacecraft is initially at $(0.05,-0.1,0)^{T}$ rad, with zero angular rates, while all the initial conditions for the second spacecraft are zero.

The synchronization gain of $\mathbf{s}_{1}$ and $\mathbf{s}_{2}$ corresponds to $\mathbf{K}_{1}+\mathbf{K}_{2}=400 \mathbf{I}$, which is larger than the tracking convergence gain $\mathbf{K}_{1}-\mathbf{K}_{2}=200 \mathbf{I}$. As a result, we can see, in Figure 4, that the first and second spacecraft exponentially synchronize first. Then, they exponentially converge together to the desired trajectory.

In order to compare the effectiveness of the exponential tracking, a simple Proportional and Derivative (PD) diffusive coupling, introduced in Ref. 6,7, is simulated for the comparison purpose, as shown in Figure 4(b). The control law for two spacecraft can be given as

$$
\begin{aligned}
& \tau_{1}=-\mathbf{K}_{1}\left(\dot{\mathbf{q}}_{1}+\Lambda \tilde{\mathbf{q}}_{1}\right)+\mathbf{K}_{2}\left(\dot{\mathbf{q}}_{2}+\Lambda \tilde{\mathbf{q}}_{2}\right) \\
& \tau_{2}=-\mathbf{K}_{1}\left(\dot{\mathbf{q}}_{2}+\Lambda \tilde{\mathbf{q}}_{2}\right)+\mathbf{K}_{2}\left(\dot{\mathbf{q}}_{1}+\Lambda \tilde{\mathbf{q}}_{1}\right)
\end{aligned}
$$

whose global asymptotic stability with respect to a constant reference input is proven in Ref. 6, 7. For a fair comparison, we selected the PD gains such that the level of control efforts is comparable to that of nonlinear control approach in (18). As shown in Figure 4(b), the PD coupling control law is not effective in following a time-varying trajectory. This is because simple linear control cannot be expected to handle the dynamic demands of efficiently following trajectories. Specifically, achieving exponential convergence ensures more effective tracking performance than asymptotic convergence by linear PD control. This is because simple linear control cannot be expected to handle the dynamic demands of efficiently following time-varying trajectories. Specifically, achieving exponential convergence ensures more effective tracking performance than asymptotic convergence by linear PD control. 

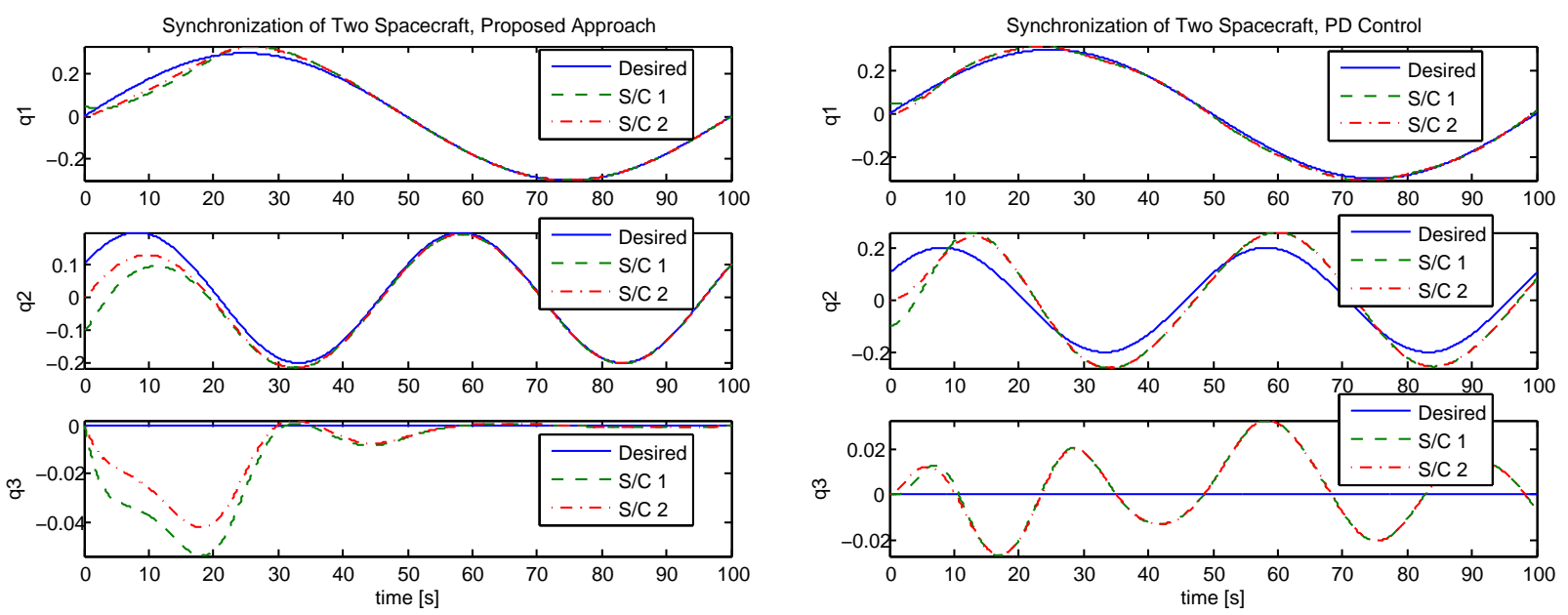

(a) Proposed control law

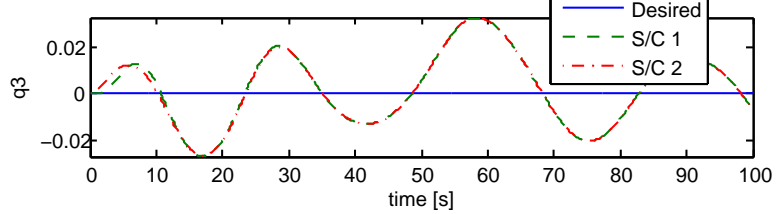

(b) Simple PD diffusive coupling

Figure 4. Synchronization of the attitude dynamics of two identical spacecraft

\section{Four Spacecraft Example}

In order to illustrate the effectiveness of the proposed approach for a larger formation, a result of simulation for four non-identical spacecraft is presented in Fig. 5. The spacecraft inertia matrices are

$$
\left[\begin{array}{ccc}
150 & 0 & -100 \\
0 & 270 & 0 \\
-100 & 0 & 300
\end{array}\right],\left[\begin{array}{ccc}
100 & 0 & -50 \\
0 & 150 & 0 \\
-50 & 0 & 250
\end{array}\right],\left[\begin{array}{ccc}
20 & 0 & -5 \\
0 & 50 & 0 \\
-5 & 0 & 65
\end{array}\right],\left[\begin{array}{ccc}
250 & 0 & -150 \\
0 & 350 & 0 \\
-150 & 0 & 400
\end{array}\right] \text { in }\left[\mathrm{kgm}^{2}\right],
$$

respectively. The desired trajectories are the same as in the previous section, and the control gains remain the same as well, such that the tracking convergence gain is $\mathbf{K}_{1}-2 \mathbf{K}_{2}=100 \mathbf{I}>0$. Figure 5 show that the four spacecraft synchronize themselves while following the desired trajectory together.
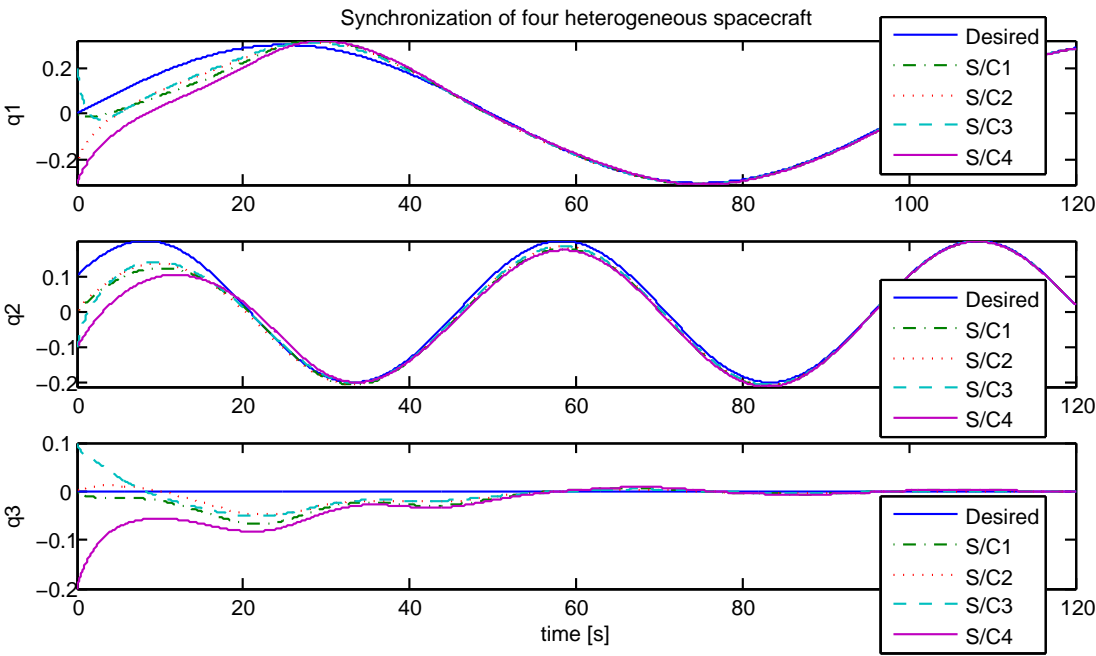

Figure 5. Synchronization of four heterogeneous spacecraft. 


\section{Effects of Communication Delays and Model Uncertainties}

Now let us discuss the robustness properties of the proposed synchronization framework. We also present a fresh perspective on adaptive synchronization that accounts for parameterized model uncertainties.

\section{A. Synchronization with Time Delays}

Wang and Slotine ${ }^{38}$ showed that contraction properties are conserved in time-delayed diffusion-like couplings. We show herein that the same principle can be applied to all the previous discussions in this paper. In particular, the proposed synchronization coupling control law in (18) is proven to synchronize multiple dynamical systems as well as to track the common trajectory, regardless of time delays in the communication.

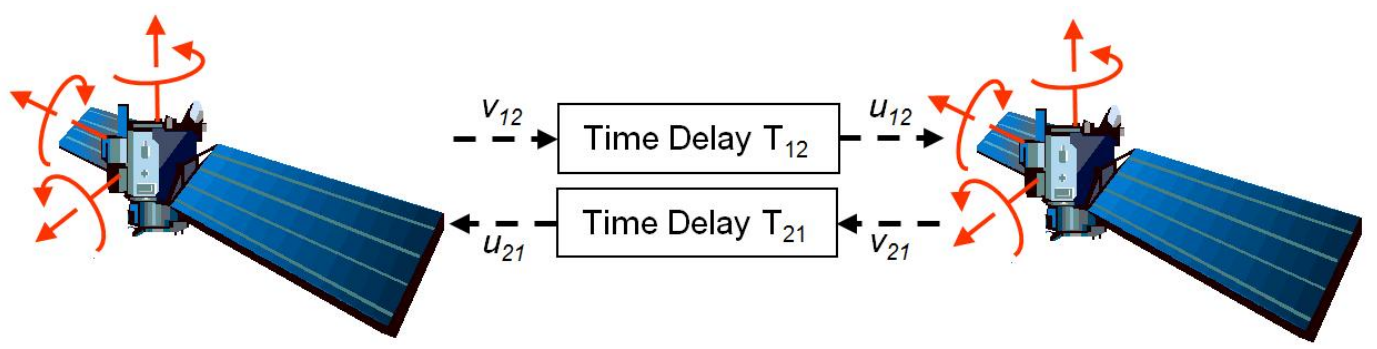

Figure 6. Synchronization of two identical spacecraft with transmission delays

Figure 6 shows two spacecraft transmitting their attitude state information to each other via time-delayed transmission channels. While $T_{12}$ is a positive constant denoting the time delay in the communication from the first spacecraft to the second spacecraft, $T_{21}$ denotes the delay from the second spacecraft to the first spacecraft. Similar to, ${ }^{38}$ we can modify our original Lagrangian systems consisting of two identical spacecraft in $(26)$ as follows

$$
\begin{aligned}
& \mathbf{M}\left(\mathbf{q}_{1}\right) \dot{\mathbf{s}}_{1}+\mathbf{C}\left(\mathbf{q}_{1}, \dot{\mathbf{q}}_{1}\right) \mathbf{s}_{1}+\left(\mathbf{K}_{1}-\mathbf{K}_{2}\right) \mathbf{s}_{1}+\mathbf{G}_{21} \tau_{21}=\mathbf{0} \\
& \mathbf{M}\left(\mathbf{q}_{2}\right) \dot{\mathbf{s}}_{2}+\mathbf{C}\left(\mathbf{q}_{2}, \dot{\mathbf{q}}_{2}\right) \mathbf{s}_{2}+\left(\mathbf{K}_{1}-\mathbf{K}_{2}\right) \mathbf{s}_{2}+\mathbf{G}_{12} \tau_{12}=\mathbf{0}
\end{aligned}
$$

where $\mathbf{G}_{21}$ and $\mathbf{G}_{12}$ are constant matrices $\left(\mathbb{R}^{n \times n}\right)$.

The communication between the two dynamics occurs by transmitting intermediate "wave" variables, defined $\operatorname{as}^{38}$

$$
\begin{array}{ll}
\mathbf{u}_{21}=\mathbf{G}_{21}^{T} \mathbf{s}_{1}+k_{21} \tau_{21} & \mathbf{v}_{12}=\mathbf{G}_{21}^{T} \mathbf{s}_{1} \\
\mathbf{u}_{12}=\mathbf{G}_{12}^{T} \mathbf{s}_{2}+k_{12} \tau_{12} & \mathbf{v}_{21}=\mathbf{G}_{12}^{T} \mathbf{s}_{2}
\end{array}
$$

where $k_{21}$ and $k_{12}$ are strictly positive constants. Time delays of $T_{21}$ and $T_{12}$ result in

$$
\mathbf{u}_{12}(t)=\mathbf{v}_{12}\left(t-T_{12}\right) \quad \mathbf{u}_{21}(t)=\mathbf{v}_{21}\left(t-T_{21}\right)
$$

Notice that the original dynamics without any communication delays are contracting since $\mathbf{K}_{1}-\mathbf{K}_{2}>0$. Expanding (55), using the above relationships on the wave variables, yields

$$
\begin{aligned}
& \mathbf{M}\left(\mathbf{q}_{1}\right) \dot{\mathbf{s}}_{1}+\mathbf{C}\left(\mathbf{q}_{1}, \dot{\mathbf{q}}_{1}\right) \mathbf{s}_{1}+\left(\mathbf{K}_{1}-\mathbf{K}_{2}\right) \mathbf{s}_{1}-\frac{1}{k_{21}} \mathbf{G}_{21}\left(\mathbf{G}_{12}^{T} \mathbf{s}_{2}\left(t-T_{21}\right)+\mathbf{G}_{21}^{T} \mathbf{s}_{1}(t)\right)=\mathbf{0} \\
& \mathbf{M}\left(\mathbf{q}_{2}\right) \dot{\mathbf{s}}_{2}+\mathbf{C}\left(\mathbf{q}_{2}, \dot{\mathbf{q}}_{2}\right) \mathbf{s}_{2}+\left(\mathbf{K}_{1}-\mathbf{K}_{2}\right) \mathbf{s}_{2}-\frac{1}{k_{12}} \mathbf{G}_{12}\left(\mathbf{G}_{21}^{T} \mathbf{s}_{1}\left(t-T_{12}\right)+\mathbf{G}_{12}^{T} \mathbf{s}_{2}(t)\right)=\mathbf{0}
\end{aligned}
$$

We can verify that (58) becomes equivalent to the original two-spacecraft dynamics in (26) by setting $k_{12}=k_{21}=1$ and $\mathbf{G}_{12}=\mathbf{G}_{12}=\sqrt{\mathbf{K}_{2}}$. Note that $\sqrt{\mathbf{K}_{2}}$ is the Cholesky decomposition of the positivedefinite symmetric matrix, $\mathbf{K}_{2}$. 
The resultant equations reflecting the time-delayed transmissions become

$$
\begin{aligned}
& \mathbf{M}\left(\mathbf{q}_{1}\right) \dot{\mathbf{s}}_{1}+\mathbf{C}\left(\mathbf{q}_{1}, \dot{\mathbf{q}}_{1}\right) \mathbf{s}_{1}+\mathbf{K}_{1} \mathbf{s}_{1}-\mathbf{K}_{2} \mathbf{s}_{2}\left(t-T_{21}\right)=\mathbf{0} \\
& \mathbf{M}\left(\mathbf{q}_{2}\right) \dot{\mathbf{s}}_{2}+\mathbf{C}\left(\mathbf{q}_{2}, \dot{\mathbf{q}}_{2}\right) \mathbf{s}_{2}+\mathbf{K}_{1} \mathbf{s}_{2}-\mathbf{K}_{2} \mathbf{s}_{1}\left(t-T_{12}\right)=\mathbf{0}
\end{aligned}
$$

which can be shown to be asymptotically contracting using the following differential length similar to ${ }^{38}$

$$
V=\frac{1}{2} \delta \mathbf{s}_{1}^{T} \mathbf{M}\left(\mathbf{q}_{1}\right) \delta \mathbf{s}_{1}+\frac{1}{2} \delta \mathbf{s}_{2}^{T} \mathbf{M}\left(\mathbf{q}_{2}\right) \delta \mathbf{s}_{2}+\frac{1}{2} V_{1,2}
$$

where

$$
V_{1,2}=\int_{t-T_{12}}^{t} \delta \mathbf{v}_{12}^{T} \delta \mathbf{v}_{12} d \epsilon+\int_{t-T_{21}}^{t} \delta \mathbf{v}_{21}^{T} \delta \mathbf{v}_{21} d \epsilon-\int_{-T_{12}}^{0} \delta \mathbf{v}_{12}^{T} \delta \mathbf{v}_{12} d \epsilon-\int_{-T_{21}}^{0} \delta \mathbf{v}_{21}^{T} \delta \mathbf{v}_{21} d \epsilon
$$

In conclusion, the formation flying spacecraft systems, individually contracting (exponentially converging) and interacting through time-delayed diffusion-like coupling in (58) are asymptotically contracting regardless of the values of the time delays. This in turn implies that individual spacecraft still synchronize and follow the common trajectory asymptotically regardless of the time delays. We have exactly matched the analysis in Ref. ${ }^{38}$ to allow the derivation of a time-delayed version of the proposed tracking control law in this paper. This shows that the proposed control law and its closed-loop system in (21) possess some robustness properties with respect to time delays.

\section{B. Effect of Bounded Disturbances}

Equation (34), in the present of the external disturbance torque $\tau_{\text {ext }}$, can be written as

$$
\left[\mathbf{M}_{2 T}\right] \dot{\mathbf{z}}+\left[\mathbf{C}_{2 T}\right] \mathbf{z}+\left[\mathbf{D}_{2}\right] \mathbf{z}=\mathbf{V}^{T}\left(\tau_{\mathbf{e x t}, \mathbf{1}}, \tau_{\mathbf{e x t}, \mathbf{2}}\right)^{T}
$$

which indicates that the disturbance input for the synchronization is only the difference $\frac{1}{\sqrt{2}}\left(\tau_{\mathbf{e x t}, \mathbf{1}}-\tau_{\mathbf{e x t}, \mathbf{2}}\right)$. As a result, the disturbance torque that is almost invariant from spacecraft to spacecraft, does not affect the synchronization of the relative attitude, which might be of more significance than the performance of trajectory following (e.g stellar interferometers).

Now we consider the bounded vanishing disturbance of the individual tracking dynamics. Due to exponential tracking convergence of the proposed scheme, the property of robustness to bounded deterministic disturbances can easily be determined. For example, consider the closed-loop system in (23), which is now subject to a vanishing perturbation ${ }^{9}$ such that $\mathbf{g}(t, \mathbf{x}=\mathbf{0})=\mathbf{0}$ :

$$
[\mathbf{M}] \dot{\mathbf{x}}+[\mathbf{C}] \mathbf{x}+\left[\mathbf{L}_{\mathbf{K}_{1},-\mathbf{K}_{2}}^{p}\right] \mathbf{x}=\mathbf{g}(t, \mathbf{x})
$$

The perturbation term $\mathbf{g}(t, \mathbf{x})$ vanishes at the equilibrium manifold $\mathbf{x}=\mathbf{0}$. Let us further assume that it satisfies the linear growth bound such that

$$
\|\mathbf{g}(t, \mathbf{x})\| \leq \gamma\|\mathbf{x}\|, \quad \forall t>0 .
$$

where $\gamma$ is a positive constant.

The squared-length analysis yields

$$
\begin{aligned}
\frac{d}{d t}\left(\delta \mathbf{x}^{T}[\mathbf{M}] \delta \mathbf{x}\right) & =2 \delta \mathbf{x}^{T}[\mathbf{M}] \delta \dot{\mathbf{x}}+\delta \mathbf{x}^{T}[\dot{\mathbf{M}}] \delta \mathbf{x} \\
& =2 \delta \mathbf{x}^{T}\left(-[\mathbf{C}] \delta \mathbf{x}-\left[\mathbf{L}_{\mathbf{K}_{1},-\mathbf{K}_{2}}^{p}\right] \delta \mathbf{x}+\delta \mathbf{g}(t, \mathbf{x})\right)+\delta \mathbf{x}^{T}[\dot{\mathbf{M}}] \delta \mathbf{x} \\
& \leq-2 \delta \mathbf{x}^{T}\left[\mathbf{L}_{\mathbf{K}_{1},-\mathbf{K}_{2}}^{p}\right] \delta \mathbf{x}+2 \gamma \delta \mathbf{x}^{T} \delta \mathbf{x}
\end{aligned}
$$

where we used the skew-symmetric property of $[\dot{\mathbf{M}}]-2[\mathbf{C}]$.

Hence, the closed-loop system in (63) is contracting in the presence of the bounded disturbance if $\left\|\left[\mathbf{L}_{\mathbf{K}_{1},-\mathbf{K}_{2}}^{p}\right]\right\|>\gamma$. This condition corresponds to $\left\|\mathbf{K}_{1}-\mathbf{K}_{2}\right\|>\gamma$ for two-spacecraft systems. As a result, the tracking gain also determines how robust the closed-loop system is with respect to a bounded disturbance. For a nonvanishing perturbation such that $\|\mathbf{g}(t, \mathbf{x})\| \leq \gamma\|\| \mathbf{x} \|+\delta$, the comparison method $^{9}$ can straightforwardly be developed to derive a bound on the solution. It should be emphasized that the exponential stability of the closed-loop system facilitates such a perturbation analysis, which highlights another benefit of contraction analysis. In contrast, the proof of robustness with asymptotic convergence is more involved. ${ }^{9}$ 


\section{Adaptive Synchronization}

Consider the following adaptive control law, which has the same local coupling structure as the proposed control law in (18):

$$
\begin{aligned}
\tau_{i} & =\mathbf{Y}_{i} \hat{\mathbf{a}}_{i}-\mathbf{K}_{1} \mathbf{s}_{i}+\mathbf{K}_{2} \mathbf{s}_{i-1}+\mathbf{K}_{2} \mathbf{s}_{i+1} \\
& =\hat{\mathbf{M}}_{i} \ddot{\mathbf{q}}_{i r}+\hat{\mathbf{C}}_{i} \dot{\mathbf{q}}_{i r}+\hat{\mathbf{g}}_{i}\left(\mathbf{q}_{i}\right)-\mathbf{K}_{1} \mathbf{s}_{i}+\mathbf{K}_{2} \mathbf{s}_{i-1}+\mathbf{K}_{2} \mathbf{s}_{i+1}
\end{aligned}
$$

where $\mathbf{s}_{i}$ denotes the composite variable for the $i$-th spacecraft such that $\mathbf{s}_{i}=\dot{\mathbf{q}}_{i}-\dot{\mathbf{q}}_{i r}$. The parameter estimate $\hat{\mathbf{a}}_{i}$ for the $i$-th member is updated by the correlation integral:

$$
\dot{\hat{\mathbf{a}}}_{i}=-\Gamma \mathbf{Y}_{i}^{T} \mathbf{s}_{i}
$$

where $\boldsymbol{\Gamma}$ is a symmetric positive definite matrix. Hence, the closed-loop system for a network comprised of two non-identical spacecraft can be written as

$$
\begin{aligned}
& {\left[\begin{array}{cc}
\mathbf{M}_{1}\left(\mathbf{q}_{1}\right) & \mathbf{0} \\
\mathbf{0} & \boldsymbol{\Gamma}^{-1}
\end{array}\right]\left(\begin{array}{c}
\dot{\mathbf{s}}_{1} \\
\dot{\tilde{\mathbf{a}}}_{1}
\end{array}\right)+\left[\begin{array}{cc}
\mathbf{C}_{1}\left(\mathbf{q}_{1}, \dot{\mathbf{q}}_{1}\right)+\mathbf{K}_{1}+\mathbf{K}_{2} & -\mathbf{Y}_{1} \\
\mathbf{Y}_{1}^{T} & \mathbf{0}
\end{array}\right]\left(\begin{array}{c}
\mathbf{s}_{1} \\
\tilde{\mathbf{a}}_{1}
\end{array}\right)=\left(\begin{array}{c}
\mathbf{u}(t) \\
\mathbf{0}
\end{array}\right)} \\
& {\left[\begin{array}{cc}
\mathbf{M}_{2}\left(\mathbf{q}_{2}\right) & \mathbf{0} \\
\mathbf{0} & \boldsymbol{\Gamma}^{-1}
\end{array}\right]\left(\begin{array}{c}
\dot{\mathbf{s}}_{2} \\
\dot{\tilde{\mathbf{a}}}_{2}
\end{array}\right)+\left[\begin{array}{cc}
\mathbf{C}_{2}\left(\mathbf{q}_{2}, \dot{\mathbf{q}}_{2}\right)+\mathbf{K}_{1}+\mathbf{K}_{2} & -\mathbf{Y}_{2} \\
\mathbf{Y}_{2}^{T} & \mathbf{0}
\end{array}\right]\left(\begin{array}{c}
\mathbf{s}_{2} \\
\tilde{\mathbf{a}}_{2}
\end{array}\right)=\left(\begin{array}{c}
\mathbf{u}(t) \\
\mathbf{0}
\end{array}\right)}
\end{aligned}
$$

where $\mathbf{u}(t)=\mathbf{K}_{2}\left(\mathbf{s}_{1}+\mathbf{s}_{2}\right)$. Additionally, $\tilde{\mathbf{a}}$ denotes an error of the estimate such that $\tilde{\mathbf{a}}=\hat{\mathbf{a}}-\mathbf{a}$. Note that $\mathbf{a}$ is a constant vector of the true parameter values, resulting in $\dot{\tilde{\mathbf{a}}}_{i}=\dot{\hat{\mathbf{a}}}_{i}$.

Similar to Section IV, applying the spectral transformation using

$$
\mathbf{V}=\left[\begin{array}{cccc}
\frac{1}{\sqrt{2}} \mathbf{I} & \frac{1}{\sqrt{2}} \mathbf{I} & \mathbf{0} & \mathbf{0} \\
\frac{1}{\sqrt{2}} \mathbf{I} & -\frac{1}{\sqrt{2}} \mathbf{I} & \mathbf{0} & \mathbf{0} \\
\mathbf{0} & \mathbf{0} & \mathbf{I} & \mathbf{0} \\
\mathbf{0} & \mathbf{0} & \mathbf{0} & \mathbf{I}
\end{array}\right]
$$

to the system, defined by the vector $\mathbf{x}=\left(\mathbf{s}_{1}, \mathbf{s}_{2}, \mathbf{a}_{1}, \mathbf{a}_{2}\right)^{T}$, leads to the following generalized Jacobian matrix

$$
\mathbf{J}=-\left[\begin{array}{cccc}
\mathbf{K}_{1}-\mathbf{K}_{2} & \mathbf{0} & -\frac{1}{\sqrt{2}} \mathbf{Y}_{1} & -\frac{1}{\sqrt{2}} \mathbf{Y}_{2} \\
\mathbf{0} & \mathbf{K}_{1}+\mathbf{K}_{2} & -\frac{1}{\sqrt{2}} \mathbf{Y}_{1} & \frac{1}{\sqrt{2}} \mathbf{Y}_{2} \\
\frac{1}{\sqrt{2}} \mathbf{Y}_{1} & \frac{1}{\sqrt{2}} \mathbf{Y}_{1} & \mathbf{0} & \mathbf{0} \\
\frac{1}{\sqrt{2}} \mathbf{Y}_{2} & -\frac{1}{\sqrt{2}} \mathbf{Y}_{2} & \mathbf{0} & \mathbf{0}
\end{array}\right]
$$

whose symmetric part indicates the system is semi-contracting with $\mathbf{K}_{1}-\mathbf{K}_{2}>0$ and $\mathbf{K}_{1}+\mathbf{K}_{2}>0$. Using Barbalat's lemma (see Section IV, Case II), it is straightforward to show that $\mathbf{s}_{1}$ and $\mathbf{s}_{2}$ tend to each other asymptotically while the parameter estimates $\mathbf{a}_{1}$ and $\mathbf{a}_{2}$ synchronize as well. This result implies that the adaptive synchronization law in (66) not only synchronizes the states of multiple dynamics in the network, but also makes the estimated physical parameters tend to each other in the presence of model uncertainties. Due to the asymptotic convergence of the adaptive control law in (66), the convergence result is now asymptotic instead of exponential.

\section{Conclusions}

We have presented the new synchronization tracking control law that can be directly applied to cooperative control of formation flying spacecraft. The proposed decentralized control law, which requires only local coupling feedback for global exponential convergence, eliminates both the all-to-all coupling and the feedback of the acceleration terms, thereby reducing communication burdens and complexity. Furthermore, in contrast with prior work which used simple single or double integrator models, the proposed method permits highly nonlinear systems such as the attitude dynamics of spacecraft. It should be noted again that one of the main contributions of this paper lies with the use of a new differential stability framework called 
contraction theory, yielding the exact proof of nonlinear stability under a variety of conditions, while many others can still come up with nonlinear control laws, similar to our approach, without a rigorous stability proof. Contraction analysis, overcoming a local result of Lypunov's indirect method, yields global results based on differential stability analysis. It has been emphasized that there exist two different time-scales in the closed-loop systems: the faster convergence rate represents the transient boundary layer dynamics of synchronization while the slower rate determines how fast the synchronized systems track the common reference trajectory. Exponential synchronization with a faster convergence rate enables reduction of multiple dynamics into a simpler form, thereby simplifying the stability analysis. It should be noted that the tracking convergence rate also determines the robustness of the closed-loop system with respect to a bounded disturbance.

The proposed bi-directional coupling has also been generalized to permit partial-state coupling, thereby further reducing communication requirements. Extensions to PD coupling, time-delayed communications, and adaptive synchronization exemplify the benefit of the differential stability analysis based on contraction theory. Simulation results show the effectiveness of the proposed control strategy for attitude tracking of spacecraft formations. For future work, we are interested in extending the proposed method to formation flying networks on unbalanced or open graphs. Robustness properties with respect to more general classes of external disturbance and sensor noise should be studied as well.

\section{ACKNOWLEDGMENTS}

The authors gratefully acknowledge anonymous reviewers' comments for the previous and current versions of the paper. Also, Soon-Jo Chung appreciates Prof. Bong Wie for stimulating discussions.

\section{APPENDIX: Contraction Theory}

We exploit partial contraction theory ${ }^{37}$ to prove the stability of coupled nonlinear dynamics. Lyapunov's linearization method indicates that the local stability of the nonlinear system can be analyzed using its differential approximation. What is new in contraction theory is that a differential stability analysis can be made exact, thereby yielding global results on the nonlinear system. A brief review of the results from ${ }^{14,30,37}$ is presented in this section. Readers are referred to these references for detailed descriptions and proofs on the following theorems. Note that contraction theory is a generalization of the classical Krasovskii's theorem. ${ }^{18}$

Consider a smooth nonlinear system

$$
\dot{\mathbf{x}}(t)=\mathbf{f}(\mathbf{x}(t), \mathbf{u}(\mathbf{x}, t), t)
$$

where $\mathbf{x}(t) \in \mathbb{R}^{n}$, and $\mathbf{f}: \mathbb{R}^{n} \times \mathbb{R}^{m} \times \mathbb{R}_{+} \rightarrow \mathbb{R}^{n}$. A virtual displacement, $\delta \mathbf{x}$ is defined as an infinitesimal displacement at a fixed time- a common supposition in the calculus of variations.

Theorem VII.1 For the system in (71), if there exists a uniformly positive definite metric,

$$
\mathbf{M}(\mathbf{x}, t)=\mathbf{\Theta}(\mathbf{x}, t)^{T} \mathbf{\Theta}(\mathbf{x}, t)
$$

where $\boldsymbol{\Theta}$ is some smooth coordinate transformation of the virtual displacement, $\delta \mathbf{z}=\boldsymbol{\Theta} \delta \mathbf{x}$, such that the associated generalized Jacobian, $\mathbf{F}$ is uniformly negative definite, i.e., $\exists \lambda>0$ such that

$$
\mathbf{F}=\left(\dot{\boldsymbol{\Theta}}(\mathbf{x}, t)+\mathbf{\Theta}(\mathbf{x}, t) \frac{\partial \mathbf{f}}{\partial \mathbf{x}}\right) \mathbf{\Theta}(\mathbf{x}, t)^{-1} \leq-\lambda \mathbf{I},
$$

then all system trajectories converge globally to a single trajectory exponentially fast regardless of the initial conditions, with a global exponential convergence rate of the largest eigenvalues of the symmetric part of $\mathbf{F}$.

Such a system is said to be contracting. The proof is given in Ref. ${ }^{14}$ Equivalently, the system is contracting if $\exists \lambda>0$ such that

$$
\dot{\mathbf{M}}+\left(\frac{\partial \mathbf{f}}{\partial \mathbf{x}}\right)^{T} \mathbf{M}+\mathbf{M} \frac{\partial \mathbf{f}}{\partial \mathbf{x}} \leq-2 \lambda \mathbf{M}
$$

It can also be shown that for a contracting autonomous system of the form $\dot{\mathbf{x}}=\mathbf{f}(\mathbf{x}, \mathbf{u}(\mathbf{x}))$, all trajectories converge to an equilibrium point exponentially fast. In essence, contraction analysis implies that stability 
of nonlinear systems can be analyzed more simply by checking the negative definiteness of a proper matrix, rather than finding some implicit motion integral as in Lyapunov theory.

The following theorems are used to derive stability and synchronization of the coupled dynamics systems.

\section{Theorem VII.2 Hierarchical combination ${ }^{30,37}$}

Consider two contracting systems, of possibly different dimensions and metrics, and connect them in series, leading to a smooth virtual dynamics of the form

$$
\frac{d}{d t}\left(\begin{array}{c}
\delta \mathbf{z}_{1} \\
\delta \mathbf{z}_{2}
\end{array}\right)=\left(\begin{array}{cc}
\mathbf{F}_{11} & \mathbf{0} \\
\mathbf{F}_{21} & \mathbf{F}_{\mathbf{2 2}}
\end{array}\right)\left(\begin{array}{c}
\delta \mathbf{z}_{1} \\
\delta \mathbf{z}_{2}
\end{array}\right)
$$

Then the combined system is contracting if $\mathbf{F}_{\mathbf{2 1}}$ is bounded.

\section{Theorem VII.3 Partial contraction ${ }^{37}$}

Consider a nonlinear system of the form $\dot{\mathbf{x}}=\mathbf{f}(\mathbf{x}, \mathbf{x}, t)$ and assume that the auxiliary system $\dot{\mathbf{y}}=\mathbf{f}(\mathbf{y}, \mathbf{x}, t)$ is contracting with respect to $\mathbf{y}$. If a particular solution of the auxiliary $\mathbf{y}$-system verifies a specific smooth property, then all trajectories of the original x-system verify this property exponentially. The original system is said to be partially contracting.

\section{Theorem VII.4 Synchronization ${ }^{37}$}

Consider two coupled systems. If the dynamics equations verify

$$
\dot{\mathbf{x}}_{1}-\mathbf{f}\left(\mathbf{x}_{1}, t\right)=\dot{\mathbf{x}}_{2}-\mathbf{f}\left(\mathbf{x}_{2}, t\right)
$$

where the function $\mathbf{f}(\mathbf{x}, t)$ is contracting in an input-independent metric, then $\mathbf{x}_{1}$ and $\mathbf{x}_{2}$ will converge to each other exponentially, regardless of the initial conditions. Mathematically, stable concurrent synchronization corresponds to convergence to a flow-invariant linear subspace of the global state space. ${ }^{26}$

\section{References}

\footnotetext{
${ }^{1}$ Ahsun, U., "Dynamics and Control of Electromagnetic Satellite Formations," Ph.D. dissertation, Department of Aeronautics and Astronautics, MIT, June 2007.

${ }^{2}$ Chopra, N., and Spong, M.W., "On Synchronization of Networked Passive Systems with Applications to Bilateral Teleoperation," Society of Instrumentation and Control Engineering of Japan Annual Conference, Okayama, Japan, August 8-10, 2005.

${ }^{3}$ Chung, F., Spectral Graph Theory, Number 92 in CBMS Regional Conference Series in Mathematics, American Mathematical Society, 1997.

${ }^{4}$ Chung, S.-J., Miller, D.W., deWeck, O.L., "ARGOS Testbed: Study of Multidisciplinary Challenges of Future Spaceborne Interferometric Arrays," SPIE Optical Engineering, Vol. 43, No. 9, pp. 2156-2167, September 2004.

${ }^{5}$ Chung, S.-J., Slotine, J.-J.E., and Miller, D.W., "Nonlinear Model Reduction and Decentralized Control of Tethered Formation Flight," to appear in Journal of Guidance, Control, and Dynamics, 2007.

${ }^{6}$ Chung, S.-J., and Slotine, J.-J.E, "Cooperative Robot Control and Synchronization of Lagrangian Systems," International Journal of Robotics Research, submitted

${ }^{7}$ Chung, S.-J., Nonlinear Control and Synchronization of Multiple Lagrangian Systems with Application to Tethered Formation Flight Spacecraft, Doctor of Science thesis, Department of Aeronautics and Astronautics, MIT, June 2007.

${ }^{8}$ Jadbabaie, A., Lin, J., and Morse, A. S., "Coordination of Groups of Mobile Autonomous Agents Using Nearest Neighbor Rules," IEEE Transactions on Automatic Control, May 2003.

${ }^{9}$ Khalil, H.K., Nonlinear Systems, 3rd Ed.,Prentice Hall, Upper Saddle River, NJ, 2002.

${ }^{10}$ Lawton, J. and Beard, R.W., "Synchronized Multiple Spacecraft Rotations," Automatica, Vol. 38, No. 8, pp. 1359-1364, 2002.

${ }^{11}$ Lee, D. and Li, P.Y., "Formation and Maneuver Control of Multiple Spacecraft ," Proceedings of the 2003 American Control Conference, Vol.1, pp. 278 - 283, June 2003.

${ }^{12}$ Lee, D. and Spong, M.W., "Stable Flocking of Multiple Inertial Agents on Balanced Graph," Proceedings of the 2006 American Control Conference, June 2006.

${ }^{13}$ Leonard, N.E., and Fiorelli, E., "Virtual Leaders, Artificial Potentials and Coordinated Control of Groups," 40th IEEE Conference on Decision and Control, 2001.

${ }^{14}$ Lohmiller, W., and Slotine, J.J.E., "On Contraction Analysis for Nonlinear Systems," Automatica, 34(6), 1998.

${ }^{15}$ Lohmiller, W., and Slotine, J.J.E., "High-Order Nonlinear Contraction Analysis," MIT NSL Report, NSL-050901.

${ }^{16}$ Mesbahi, M., and Hadaegh, F.Y., "Formation Flying of Multiple Spacecraft via Graphs, Matrix Inequalities, and Switching," AIAA Journal of Guidance, Control, and Dynamics, (24) 2: 369-377, 2001.

${ }^{17}$ Slotine, J.J.E. and Benedetto, M.D.D., "Hamiltonian Adaptive Control of Spacecraft," IEEE Transactions on Automatic Control, Vol. 35, No. 7, July 1990, pp. 848-852.
} 
${ }^{18}$ Slotine, J.-J.E., and Li, W., Applied Nonlinear Control, Prentice Hall,. New Jersey, 1991.

${ }^{19}$ Wie, Bong, Space Vehicle Dynamics and Control, 2nd ed., AIAA, Reston, Virginia, 2007.

${ }^{20}$ Yoon, H., and Tsiotras, P., "Spacecraft Adaptive Attitude and Power Tracking with Variable Speed Control Moment Gyroscopes," AIAA Journal of Guidance, Control, and Dynamics, Vol. 25, No. 6, pp. 1081-1090, November-December 2002. 1993.

${ }^{21}$ Shuster, M.D., "A Survey of Attitude Representations," Journal of the Astronautical Sciences, Vol. 41, No. 4, pp. 439-517,

${ }^{22}$ Schaub, H., and Junkins, J. L, "Stereographic Orientation Parameters for Attitude Dynamics: A Generalization of the Rodrigues Parameters," Journal of the Astronautical Sciences, Vol. 44, No. 1, pp. 1-19, Jan.-Mar. 1993.

${ }^{23}$ Olfati-Saber, R., and Murray, R.M., "Consensus Problems in Networks of Agents with Switching Topology and TimeDelays," in the special issue of the IEEE Transactions On Automatic Control on Networked Control Systems, VOL. 49, NO. 9, Sept. 2004.

${ }^{24}$ Ögren, P., Egerstedt, M. and Hu, X., "A Control Lyapunov Function Approach to Multiagent Coordination," IEEE Transactions on Robotics and Automation, Vol. 18, No. 5, October 2002.

${ }^{25}$ Ögren, P., Fiorelli, E., and Leonard, N.E., "Cooperative Control of Mobile Sensor Networks: Adaptive Gradient Climbing in a Distributed Environment," IEEE Trans. on Automatic Control, 49(8):1292.1302, 2004.

${ }^{26}$ Pham, Q.-C., and Slotine, J.J. E., "Stable Concurrent Synchronization in Dynamic System Networks," Neural Networks, 20(1), 2007.

${ }^{27}$ Pan, H., and Kapila, V., "Adaptive Nonlinear Control for Spacecraft Formation Flying with Coupled Translational and Attitude Dynamics," Proc. of the 40th IEEE Conference on Decision and Control, Orlando, FL, December 2001.

${ }^{28}$ Ploen, S.R., Hadaegh, F.Y., and Scharf, D.P., "Ridgid Body Equations of Motion for Modeling and Control of Spacecraft Formations - Part 1: Absolute Equations of Motion," Proc. of the 2004 American Control Conference, Boston, 2004.

${ }^{29}$ Rodriguez-Angeles, A., and Nijmeijer, H., "Mutual Synchronization of Robots via Estimated Sate Feedback: A Cooperative Approach," IEEE Transactions on Control Systems Technology, Vol. 12, No. 4, 2004. .

${ }^{30}$ Slotine, J.-J.E., "Modular Stability Tools for Distributed Computation and Control," Int. J. Adaptive Control and Signal Processing, 17(6), 2003.

${ }^{31}$ Slotine, J.-J.E., and Lohmiller, W., "Modularity, Evolution, and the Binding Problem: A View from Stability Theory," Neural Networks, 14(2), 2001.

${ }^{32}$ Strang, G., Introduction to Applied Mathematics, Wellesley-Cambridge Press, Wellesey, MA, 1986.

${ }^{33}$ Scharf, D.P., Hadaegh, F.Y., and Ploen, S.R., "A Survey of Spacecraft Formation Flying Guidance and Control (Part I): Guidance," Proceedings of the American Control Conference, Denver, CO, June 4-6,2003.

${ }^{34}$ Scharf, D.P., Hadaegh, F.Y., and Ploen, S.R., "A Survey of Spacecraft Formation Flying Guidance and Control (Part II): Control," Proceedings of the American Control Conference, Denver, CO, June 4-6,2003.

${ }^{35}$ Vidyasagar, M., Nonlinear Systems Analysis, 2nd ed., SIAM Classics in Mathematics, SIAM, Philadelphia, 1993.

${ }^{36}$ Wang, P.K.C., Hadaegh, F.Y., and Lau, K., "Synchronized Formation Rotation and Attitude Control of Multiple FreeFlying Spacecraft," Journal of Guidance, Control, and Dynamics, Vol. 22, No. 1, 1999.

${ }^{37}$ Wang, W., and Slotine, J.J.E., "On Partial Contraction Analysis for Coupled Nonlinear Oscillators," Biological Cybernetics, 92(1). 2004.

${ }^{38}$ Wang, W., and Slotine, J.J.E., "Contraction Analysis of Time-Delayed Communications Using Simplified Wave Variables," IEEE Trans. Aut. Control, 51(5), 2006. 